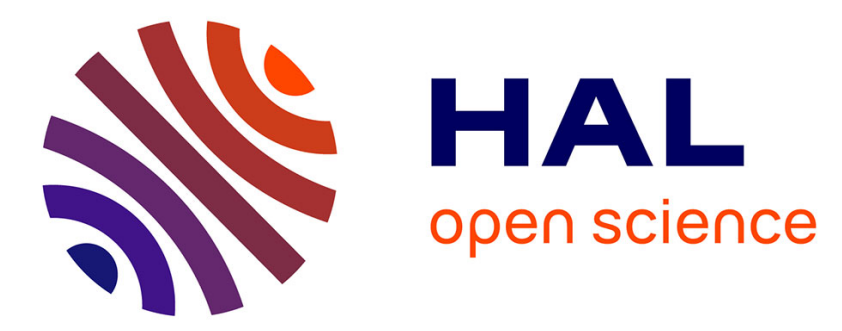

\title{
Dynamic Gains Differentiator for Hydraulic System Control
}

\author{
Lilia Sidhom, Xavier Brun, Mohamed Smaoui, Eric Bideaux, Daniel \\ Thomasset
}

\section{- To cite this version:}

Lilia Sidhom, Xavier Brun, Mohamed Smaoui, Eric Bideaux, Daniel Thomasset. Dynamic Gains Differentiator for Hydraulic System Control. Journal of Dynamic Systems, Measurement, and Control, 2015, 137 (4), pp.DS-13-1027. 10.1115/1.4029271 . hal-01153973

\section{HAL Id: hal-01153973 \\ https://hal.science/hal-01153973}

Submitted on 23 Apr 2019

HAL is a multi-disciplinary open access archive for the deposit and dissemination of scientific research documents, whether they are published or not. The documents may come from teaching and research institutions in France or abroad, or from public or private research centers.
L'archive ouverte pluridisciplinaire HAL, est destinée au dépôt et à la diffusion de documents scientifiques de niveau recherche, publiés ou non, émanant des établissements d'enseignement et de recherche français ou étrangers, des laboratoires publics ou privés.

\section{다)(1) $(5$}

Distributed under a Creative Commons Attribution - NonCommercial| 4.0 International 


\title{
Dynamic Gains Differentiator For Hydraulic System Control
}

\author{
L. Sidhom, X. Brun, M. Smaoui, E. Bideaux and D. Thomasset
}

\begin{abstract}
This paper deals with online numerical differentiation of a noisy time signal where new higher order sliding modes differentiators are proposed. The key point of these algorithms is to include a dynamic on the differentiator parameters. These dynamics tune-up automatically the algorithm gains in real time. Convergence properties of the new schemes are derived using a Lyapunov approach. Their effectiveness is illustrated via simulations and experimental tests, where comparative studies are performed between classical schemes and the new ones. Such algorithms are also used in the feedback control of an electrohydraulic system.
\end{abstract}

Index Terms-Differentiator/Controller design, Higher order sliding modes, Dynamic gains, Electro-hydraulic system.

\section{INTRODUCTION}

$\mathbf{T}$ He purpose of this paper is to propose a new approach to design a suitable scheme to estimate the derivatives of a measured signal. It is well known that the main challenge is to have a large bandwidth for the differentiator response while avoiding noise corruption of the signal. These problems come from the limited information provided by the measured signal. Therefore, the problem is to find a suitable linear or nonlinear differentiation algorithm able to reduce noise effect while leaving the informative signal unchanged without phase shift. Actually, the ideal differentiator design is a well-known issue but remains a difficult to achieve. Therefore, the differentiator that we are looking for should be able to reduce noise effect while leaving the informative signal unchanged without phase shift.

There are basically two major approaches to estimate the signal derivatives. In the first approach, the synthesis of differentiators requires a good knowledge of the system model. In this case the differentiator design is reduced to an observation problem. In [24], [25], the proposed differentiator is a high gain observer, that is a Luenberger state observer with particular pole placement. However, the differentiator parameters are not tuned to reduce sensitivity to measurement noises or perturbations. The case of random noises/perturbations (such as Gaussian white noises) is addressed by Kalman observers whose gains are computed by the resolution of an algebraic Ricatti equation, [26]. The high gain differentiators in [38], [39], [40] provide an exact derivative provided their gains tend to infinity which also leads to higher sensitivity to small high-frequency noises. Another drawback of the high-gain differentiators is their peaking effect. Another example for state estimation is

\footnotetext{
Laboratoire Ampère, UMR CNRS 5005, Université de Lyon, INSA-Lyon, F-69621 Villeurbanne Cedex, France lilia.sidhomeinsa-lyon.fr
}

based on nonlinear observer theory such as a backstepping observers [9]. Unfortunately, the lack of information or an insufficient knowledge on the system dynamics makes the implementation of the linear or nonlinear state observers difficult. To overcome this problem, some researches looked at the synthesis of robust observers taking into account parametric uncertainties, such as sliding mode observer [27], [28] which gave an interesting results. In [27], the super-twisting algorithm was modified in order to be used as an observer of velocity for uncertain mechanical systems. In [28], the authors proposed an approach based on the sliding mode observer to finite-time state estimation for a class of non-linear systems by taking into account the modelling uncertainties and unknown inputs.

For the second approach, the design of a differentiator is unavoidable. To build it, some features about the signal and the noise should be known. However, in many cases the structure of the signal is unknown except from some differential inequalities. In this case, there are standard approaches to compute derivative numerically such as the finite difference operators. Such approaches are easy to implement and are not computationally expensive but they present bad noise suppression capabilities. Other one, the approaches that are based on a suitable truncated Taylor expansion of the signal to differentiate [35], [36], are potentially interesting. Due to the use of iterated integrals of the observation signal, these algebraic methods exhibit good robustness properties with respect to noises. However, the results provided by such methods are sensitive to the truncation order also to the size of the sliding window and the setting of other parameters. Alternative approaches based on the sliding mode technique can also be used [10]. A possible choice is to implement a sliding mode differentiator, as the one proposed by Levant [14], [12]. In this approach, the author uses an arbitrary-order robust differentiator with finite-time convergence, which it has a simple form and is therefore easy to be implemented. According to [12], 1storder and 2nd-order differentiator can be defined employing this approach. The major problem associated with these algorithms is the tuning of its gains to match convergence and good performance. The accuracy of these differentiators depends on the choice of these parameters. In fact, these parameters depend on the Lipschitz constant of the signal derivative. This constant is usually not known accurately beforehand, especially if the signal is noisy. To avoid this problem, different research works were proposed to modify the classical 1st-order sliding mode differentiator (super-twisting). For example, a new adaptive sliding mode 
controller based on super-twisting which is proposed to reduce the chattering phenomenon [32], [31]. In this work, the authors synthesized this approach for nonlinear systems with bounded uncertainties/perturbations whose bounds are unknown. In [30], a new scheme of the super-twisting algorithm is proposed with adding linear components. By using a Lyapunov approach, finite time convergence of the proposed algorithm and its robustness to strong perturbation terms is proved. In [29], a variable gain super-twisting algorithm is proposed which is defined to compensate some classes of uncertainties/disturbances. In [37], the authors propose also a new scheme of super-twisting differentiator. Here, the idea is to adapt the gains only when the estimation error unacceptably deviates from zero. The proposed scheme is used to estimate the rod speed of an actuator in order to detect the fault. To validate this algorithm, only simulations have been performed on a nonlinear aircraft benchmark model. In [33], [34], an adaptive sliding mode algorithm is also applied for other kinds of applications.

For many years, research efforts have been focused on the development of control laws, especially for fluid power systems. Most of them were in the field of feedback linearization [2], [3]. Other investigations have been conducted on adaptive control [4], [5] backstepping control [6], [7] and others are based on sliding mode technique [41], [42]. All of the aforementioned controllers generally require velocity and acceleration measurements to perform the feedback loop. However, accelerometers are seldom used in practical drive systems. Their inclusion increases cost, energy consumption, system complexity (the accelerometer is mounted to the load in displacement) and also reduce the system reliability. In order to avoid these drawbacks, such sensors can be replaced by a signal estimator on the closed-loop control system. However, the estimated signal can lead to a noisy and an inaccurate or delayed signal of the control law. In fact, the quality of the estimation signals is quite important to perform a control requirement.

The major contributions of this paper are the following:

(i) A new scheme of 1st-order sliding mode differentiator is proposed which is different to those already proposed in [33], [34].

(ii) A 2nd-order sliding mode differentiator is proposed which has not yet been studied in previous research.

(iii) The basic idea of these proposed algorithms is to include a dynamic law to the gains of the basic algorithms [12] and add a new linear terms on equations algorithm. These tuning laws depend on the sliding surfaces.

(iv) The proposed solution allows an online self-tuning gains of the algorithms while providing a good compromise between accuracy and robustness toward noises as compared to the basic differentiators. This last one is the major problem for most online differentiation methods.

This paper is organized as follows. In the next section, the problem of sliding mode differentiators (1st and 2nd order) is explained and illustrated with some simulation tests. In section 3, new schemes of algorithms are presented. Section 4 describes the proposed model of the electro-hydraulic test bench. The last section is devoted to experimental results obtained when the proposed differentiators are included on the control loop feedback.

\section{Differentiators Design via Sliding Mode}

\section{A. Problem Statement}

Considered as an approach that is mainly used to design one of the robust controller, sliding mode technique is also employed for differentiator design problem [12]. With using the main features of such technique, good results are obtained for the differentiation problem.

Let input signal $f(t)$ be a function defined on $[0, \infty[$ and measurable in Lebesgues sense. Regarded as the input signal of differentiator, $f(t)$ is described as the sum of the two following terms:

$$
f(t)=f_{0}(t)+\xi(t)
$$

where $f_{0}(t)$ is an unknown base signal with the $(1+n)^{t h}$ derivative having a known Lipschitz constant $C>0$, and $\xi(t)$ is a bounded Lebesgue-measurable noise with unknown features, defined by: $|\xi(t)|<\epsilon$, with $\epsilon$ sufficiently small.

With infinite number of differentiator scheme proposed in [12], the aim is to estimate in real time $\dot{f}_{0}(t), \ddot{f}_{0}(t), \cdots, f_{0}^{(n)}(t)$. These estimates are exact in the absence of noises. This scheme is defined by the following equations:

$$
\left\{\begin{array}{l}
\dot{z}_{0}=v_{0} \\
v_{0}=-\lambda_{0}\left|z_{0}-f\right|^{\frac{n}{(n+1)}} \operatorname{sign}\left(z_{0}-f\right)+z_{1} \\
\dot{z}_{1}=v_{1} \\
v_{1}=-\lambda_{1}\left|z_{1}-v_{0}\right|^{\frac{(n-1)}{n}} \operatorname{sign}\left(z_{1}-v_{0}\right)+z_{2} \\
\cdot \\
\dot{z}_{n-1}=v_{n-1} \\
v_{n-1}=-\lambda_{n-1}\left|z_{n-1}-v_{n-2}\right|^{\frac{1}{2}} \operatorname{sign}\left(z_{n-1}-v_{n-2}\right)+z_{n} \\
\dot{z}_{n}=-\lambda_{n} \operatorname{sign}\left(z_{n}-v_{n-1}\right)=-\lambda_{n} \operatorname{sign}\left(z_{n-1}-v_{n-2}\right)
\end{array}\right.
$$

where $\lambda_{i}, i \in\{0, \cdots, n\}$ are positive gains depending on the Lipschitz constant $C$ of $f_{0}^{(n+1)}(t)$. Here $z_{i}=v_{i-1}, i \in$ $\{1, \cdots, n\}$ are the outputs of the differentiator. At time $t=0$, the initial values $z_{0}(0)=f(0), z_{i}(0)=v_{i-1}(0)$ are taken, with $i \in\{1, \cdots, n\}$. The parameters $\lambda_{i}, i \in\{0, \cdots, n\}$ can be chosen by using the following expression (see the proof in [12]):

$$
\lambda_{i}=\lambda_{i 0} C^{\frac{1}{n-i+1}}
$$

Where $\lambda_{i 0}$ is positive constant. According to this expression, the gains choice assumes the knowledge in advance of the Lipschitz constant of the $(n+1)^{t h}$ derivative of the useful signal, [15]. But, if the signal is noisy, the Lipschitz constant 
is a priori unknown and the choice becomes even more difficult.

Therefore, the main drawback of such algorithm is the setting of its gains to keep good performances even when the frequency of the input signal of differentiator changes or if the input spectrum has rich frequencies. It is not always easy to tune the parameters values $\lambda_{i}$ for a given bandwidth of the input signal. A simple modification of the spectral content of the input signal or of its magnitude can greatly affect the estimation of the derivative. Usually, the gain values are found by guessing with computer simulations.

From (2), it can be seen that the terms $z_{i}$ introduce the integral components and act as estimators of the input signal derivative. Theoretically, the ideal sliding mode must ensure the first terms of equations (2) to zero in finite time. However, the ideal sliding mode never be realized in practice owing to the different origin of inaccuracy, such as a measurement errors. Moreover, the presence of "sign(.)" function in these terms leads to high frequency oscillations. Indeed, this chattering effect can deteriorate the precision of the estimated signal. Therefore, it is not easy to adjust the gains to reach a good compromise between accuracy and robustness to noise ratio.

In this paper, a 1st and a 2nd-order sliding mode differentiator are studied. From (2), the super-twisting algorithm $(n=1)$ is defined by the following equations:

$$
\left\{\begin{array}{l}
\dot{z}_{0}=v_{0} \\
v_{0}=-\lambda_{0}\left|z_{0}-f\right|^{\frac{1}{2}} \operatorname{sign}\left(z_{0}-f\right)+z_{1} \\
\dot{z}_{1}=v_{1} \\
\dot{z}_{1}=-\lambda_{1} \operatorname{sign}\left(z_{1}-v_{0}\right)=-\lambda_{1} \operatorname{sign}\left(z_{0}-v_{1}\right)
\end{array}\right.
$$

For $n=2$, a 2nd-order differentiator can be easily defined where the system 2 is rewritten as:

$$
\left\{\begin{array}{l}
\dot{z}_{0}=v_{0}, \\
v_{0}=-\lambda_{0}\left|z_{0}-f\right|^{\frac{2}{3}} \operatorname{sign}\left(z_{0}-f\right)+z_{1} \\
\dot{z}_{1}=v_{1}, \\
v_{1}=-\lambda_{1}\left|z_{1}-v_{0}\right|^{\frac{1}{2}} \operatorname{sign}\left(z_{1}-v_{0}\right)+z_{2} \\
\dot{z}_{2}=-\lambda_{2} \operatorname{sign}\left(z_{2}-v_{1}\right)=-\lambda_{2} \operatorname{sign}\left(z_{1}-v_{0}\right)
\end{array}\right.
$$

After a finite time convergence and without any noises, $z_{1}=$ $v_{0}$ is the estimation of $\dot{f}_{0}(t)$, which represents the output of the 1st-order differentiator. The second output $\ddot{f}_{0}(t)$ of the 2nd-order algorithm is given by $z_{2}=v_{1}$.

\section{B. Problem Illustration}

To investigate the influence of the choice parameters on the algorithm accuracy, simulation tests are performed for the 2nd-order sliding mode differentiator. For the 1st-test, the considered input signal is given by $g(t)=\sin (\pi t)$, where its frequency is $f=0.5 \mathrm{~Hz}$. Afterwards, this frequency is changed up to $2 \mathrm{~Hz}$, without changing the gain values of algorithm. So in this 2nd-test, the Lipschitz constant $C$ is just increased. This constant is calculated by $C=2 \pi f A$, where $A$ is the magnitude of $g(t)$. With the difference between the estimated derivative of $g(t)$ and the analytical one, the error is computed in order to compare its value for each test. The figure 1 shows the errors function of time. These errors are greatly increased when the frequency of the input signal has increased. Thus, the magnitude precision is significantly degraded.

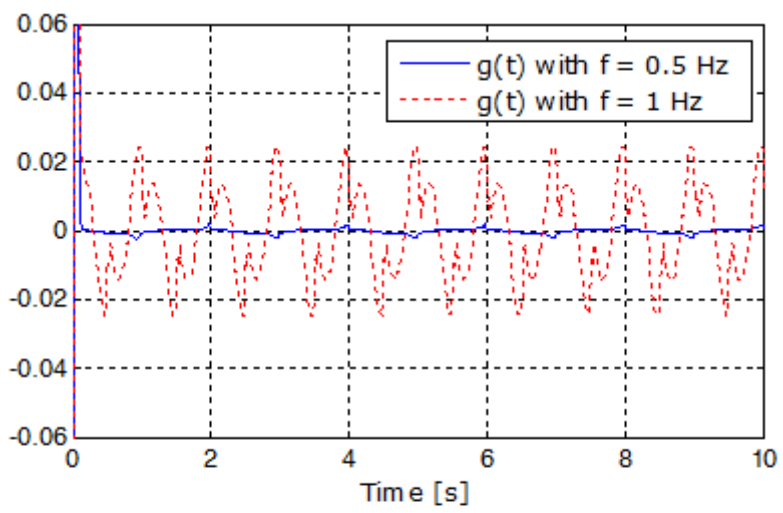

(a) Error : 1st-derivative

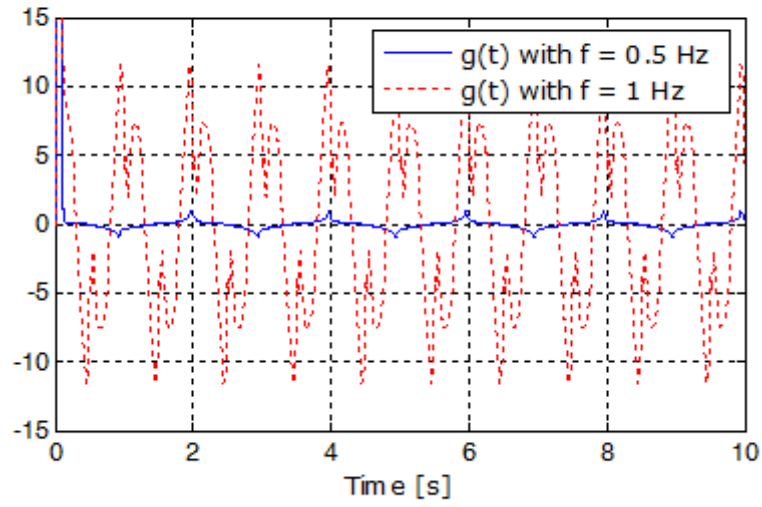

(b) Error : 2nd-derivative

Fig. 1: Errors with different frequency of $g(t)$

\section{Proposed Differentiators}

\section{A. New scheme of 1st-Order Differentiator}

The proposed 1st-order differentiator is defined by the following system:

$$
\left\{\begin{array}{l}
\dot{z_{0}}=v_{0} \\
v_{0}=-\hat{\lambda}_{0}\left|z_{0}-f\right|^{\frac{1}{2}} \operatorname{sign}\left(z_{0}-f\right)-K_{0}\left(z_{0}-f\right)+z_{1} \\
\dot{z_{1}}=v_{1} \\
v_{1}=-\hat{\lambda}_{1} \operatorname{sign}\left(z_{0}-f\right)
\end{array}\right.
$$

The sliding surfaces are given by:

$$
\left\{\begin{array}{l}
\sigma_{0}=z_{0}-f \\
\sigma_{1}=z_{1}-\dot{f}
\end{array}\right.
$$


Taking into account the variables which are defined in (7), the system (6) can be rewritten as follows:

$$
\left\{\begin{array}{l}
\dot{\sigma}_{0}=-\hat{\lambda}_{0}\left|\sigma_{0}\right|^{\frac{1}{2}} \operatorname{sign}\left(\sigma_{0}\right)-K_{0} \sigma_{0} \\
\dot{\sigma}_{1}=-\hat{\lambda}_{1} \operatorname{sign}\left(\sigma_{0}\right)
\end{array}\right.
$$

Theorem 3.1: For $K_{0}>0$ and with the sliding surfaces defined by (7), all trajectories of the algorithm (6) converge at a finite time to the equilibrium point $\sigma_{0}=\sigma_{1}=0$, with the dynamic gains $\hat{\lambda}_{i}, i \in\{0,1\}$ defined by :

$$
\left\{\begin{array}{l}
\dot{\hat{\lambda}}_{0}=\left(\left|\lambda_{0}\right|^{\frac{1}{2}} \operatorname{sign}\left(\lambda_{0}\right)\right) \lambda_{0}, \quad \hat{\lambda}_{0}(0) \geq 0 \text { and } \dot{\hat{\lambda}}_{0}>0 \forall t>0 \\
\dot{\hat{\lambda}}_{1}=\left|\lambda_{0}\right|, \quad \hat{\lambda}_{1}(0) \geq 0 \text { and } \dot{\hat{\lambda}}_{1}>0 \forall t>0
\end{array}\right.
$$

Proof:

Referring to [30], let us introduce the quadratic Lyapunov function as follows:

$$
V_{11}(\sigma)=\xi_{1}^{T} P \xi_{1}, \quad \text { with } \sigma=\left(\sigma_{0}, \sigma_{1}\right)^{T}
$$

At $t=0$, let us note $\sigma(t=0)=\sigma_{\text {init }}=\left(\sigma_{0}(0), \sigma_{1}(0)\right)^{T}$. The vector $\xi_{1}$ is chosen such that:

$$
\xi_{1}=\left(\begin{array}{c}
\left|\sigma_{0}\right|^{\frac{1}{2}} \operatorname{sign}\left(\sigma_{0}\right) \\
\sigma_{1}
\end{array}\right)
$$

and $P \in \mathbb{R}^{(2 \times 2)}$ is a symmetric matrix given by:

$$
P=\left(\begin{array}{cc}
2 \hat{\lambda}_{1}+\frac{\Phi_{0}{ }^{2}}{2} & -\frac{\Phi_{0}}{2} \\
-\frac{\Phi_{0}}{2} & 1
\end{array}\right)
$$

where $\Phi_{0}=\hat{\lambda}_{0}+K_{0}\left|\sigma_{0}\right|^{\frac{1}{2}}$ is a positive quantity since $\hat{\lambda}_{0} \geq 0$ and $K_{0}>0$.

The matrix $P$ is a positive definite matrix, since its eigenvalues $\bar{\lambda}_{1,2}$ are positive. Indeed, the characteristic polynomial equation of $P$ is given by (13) and with $\hat{\lambda}_{1} \geq 0$, we can deduce that the eigenvalues of $P$ are a positive real.

$$
\bar{\lambda}^{2}-\left(1+2 \hat{\lambda}_{1}+\frac{\Phi_{0}^{2}}{2}\right) \bar{\lambda}+2 \hat{\lambda}_{1}+\frac{\Phi_{0}^{2}}{4}=0
$$

Note that the defined Lyapunov function is continuous everywhere but not differentiable at $\sigma_{0}=0$. Here, $V_{11}(\sigma)$ is continuously differentiable, except on the set $S_{1}=\left\{\left(\sigma_{0}, \sigma_{1}\right) \in \mathbb{R}^{(2 \times 2)} \mid \sigma_{0=0}\right\}$, the Lyapunov theorem can only be applied to the points where $V_{11}(\sigma)$ is differentiable. Then, a non-smooth version of Lyapunov's theory is needed, [17].

Note that $V_{11}(\sigma)$ is however positive definite and radially bounded :

$$
\bar{\lambda}_{\min }\{P\}\left\|\xi_{1}\right\|_{2}^{2} \leq V_{11} \leq \bar{\lambda}_{\max }\{P\}\left\|\xi_{1}\right\|_{2}^{2}
$$

where $\left\|\xi_{1}\right\|$ is the euclidean norm of $\xi_{1}$ :

$$
\left\|\xi_{1}\right\|_{2}^{2}=\left|\sigma_{0}\right|+\sigma_{1}^{2}
$$

$\bar{\lambda}_{\min }\{P\}, \bar{\lambda}_{\max }\{P\}$ are respectively the minimum and maximum eigenvalues of the matrix $P$.

The derivative of the vector $\xi_{1}$ can be expressed as below:

$$
\begin{aligned}
\dot{\xi_{1}} & =\left|\sigma_{0}\right|^{-\frac{1}{2}}\left(\begin{array}{cc}
-\frac{\Phi_{0}}{2} & \frac{1}{2} \\
-\hat{\lambda}_{1} & 0
\end{array}\right) \xi_{1} \\
& =\left|\sigma_{0}\right|^{-\frac{1}{2}} A(\sigma) \xi_{1}
\end{aligned}
$$

The time derivative of $V_{11}$ along the system trajectories is then given by:

$$
\begin{aligned}
\dot{V}_{11} & =\left|\sigma_{0}\right|^{-\frac{1}{2}} \xi_{1}^{T}\left(A^{T}(\sigma) P+P A(\sigma)\right) \xi_{1} \\
& =-\left|\sigma_{0}\right|^{-\frac{1}{2}} \xi_{1}^{T} Q(\sigma) \xi_{1}
\end{aligned}
$$

where $Q$ is:

$$
Q=\frac{\Phi_{0}}{2}\left(\begin{array}{cc}
2 \hat{\lambda}_{1}+\Phi_{0}^{2} & -\Phi_{0} \\
-\Phi_{0} & 1
\end{array}\right)
$$

To conclude on the non-negativity of $\dot{V}_{11}, Q$ has to be positive definite matrix. From its characteristic polynomial equation (20), we can deduced that the eigenvalues are positive and consequently that $Q$ is positive definite one:

$$
\bar{\lambda}^{2}-\left(1+2 \hat{\lambda}_{1}+\frac{\Phi_{0}^{2}}{2}\right) \bar{\lambda}+2 \hat{\lambda}_{1}=0
$$

From (14), we can write:

$$
\left\|\xi_{1}\right\|_{2} \leq \frac{V_{11}^{\frac{1}{2}}}{\bar{\lambda}_{\min }^{\frac{1}{2}}\{P\}}
$$

Using equations (15) and (21), we have:

$$
\left|\sigma_{0}\right|^{\frac{1}{2}} \leq\left[\left|\sigma_{0}\right|+\sigma_{1}^{2}\right]^{\frac{1}{2}} \leq \frac{V_{11}^{\frac{1}{2}}}{\bar{\lambda}_{\min }^{\frac{1}{2}}\{P\}}
$$

Therefore,

$$
\dot{V}_{11} \leq-\frac{\bar{\lambda}_{\min }^{\frac{1}{2}}\{P\}}{V_{11}^{\frac{1}{2}}} \bar{\lambda}_{\min }\{Q\}\left\|\xi_{1}\right\|_{2}^{2}
$$

Using the right inequality of the expression (14), (23) becomes:

$$
\dot{V}_{11} \leq-\frac{\bar{\lambda}_{\min }^{\frac{1}{2}}\{P\} \bar{\lambda}_{\min }\{Q\}}{\bar{\lambda}_{\max }\{P\}} V_{11}^{\frac{1}{2}}=-\delta V_{11}^{\frac{1}{2}}
$$

where $\delta=\frac{\bar{\lambda}_{\min }^{\frac{1}{2}}\{P\} \bar{\lambda}_{\min }\{Q\}}{\bar{\lambda}_{\max }\{P\}}>0$.

Let us consider now the general case of a differential equation of the following form:

$$
\dot{\chi}(t)=-\delta \chi^{\frac{1}{2}}(t), \quad \chi(0)=\chi_{0} \geq 0
$$

The solution of this equation is given by:

$$
\int_{0}^{t} \frac{\dot{\chi}}{\chi^{\frac{1}{2}}} d t=-\int_{0}^{t} \delta d t \Rightarrow \chi(t)=\left(\chi_{0}^{\frac{1}{2}}-\frac{\delta}{2} t\right)^{2}
$$

From (26), $\chi(t)$ is equal to zero at a time $t^{*}$ given by:

$$
t^{*}=\frac{2 \chi_{0}^{\frac{1}{2}}}{\delta}
$$


From (27), it follows that $V_{11}(\sigma(t))$ and $\sigma(t)$ converge to zero in a finite time $T$ given by :

$$
T=\frac{2 V_{11}^{\frac{1}{2}}\left(\sigma_{\text {init }}\right)}{\delta}
$$

\section{B. New scheme of 2nd-Order Differentiator}

Let us define now the new scheme of 2nd-order sliding modes differentiator:

$$
\left\{\begin{array}{l}
\dot{z_{0}}=v_{0} \\
v_{0}=-\hat{\lambda}_{0}\left|s_{0}\right|^{\frac{2}{3}} \operatorname{sign}\left(s_{0}\right)-K_{0} s_{0}+z_{1} \\
\dot{z_{1}}=v_{1} \\
v_{1}=-\hat{\lambda}_{1}\left|s_{1}\right|^{\frac{1}{2}} \operatorname{sign}\left(s_{1}\right)-\hat{\lambda}_{2} \int_{0}^{t} \operatorname{sign}\left(s_{1}\right) d t-K_{1} s_{1}
\end{array}\right.
$$

$K_{0}$ and $K_{1}$ are positive gains and $s_{0}, s_{1}$ are the sliding surfaces given by:

$$
\left\{\begin{array}{l}
s_{0}=z_{0}-f \\
s_{1}=z_{1}-v_{0}
\end{array}\right.
$$

The dynamic gains $\hat{\lambda}_{i}, i \in\{0,1,2\}$ are defined by:

$$
\left\{\begin{array}{l}
\dot{\hat{\lambda}}_{0}=\left[\left|s_{0}\right|^{\frac{2}{3}} \operatorname{sign}\left(s_{0}\right)\right] s_{0} \\
\dot{\hat{\lambda}}_{1}=\left[\left|s_{1}\right|^{\frac{1}{2}} \operatorname{sign}\left(s_{1}\right)\right] s_{1} \\
\dot{\hat{\lambda}}_{2}=s_{1} \int_{0}^{t} \operatorname{sign}\left(s_{1}\right) d t
\end{array}\right.
$$

Theorem 3.2: For $K_{0}, K_{1}>0$ and with the dynamic gains $\hat{\lambda}_{i}, i \in\{0,1,2\}$ defined by (31), the system trajectories (29) converge locally and asymptotically towards the equilibrium point $s_{0}=s_{1}=0$.

Definie $\lambda_{0}^{*}$ and $\lambda_{1}^{*}$, a priori unknown constants which represent an ideally tuned differentiator:

$$
\left\{\begin{array}{l}
\dot{f}=-\lambda_{0}^{*}\left|s_{0}\right|^{\frac{2}{3}} \operatorname{sign}\left(s_{0}\right)+z_{1} \\
\ddot{f}=-\lambda_{1}^{*}\left|s_{1}\right|^{\frac{1}{2}} \operatorname{sign}\left(s_{1}\right)-\lambda_{2}^{*} \int_{0}^{t} \operatorname{sign}\left(s_{1}\right) d t
\end{array}\right.
$$

Proof:

Let $\sigma_{0}=s_{0}=z_{0}-f$. With this change of coordinate, the two first equations of system (29) can be written as follows:

$$
\dot{\sigma}_{0}=-\hat{\lambda}_{0}\left|\sigma_{0}\right|^{\frac{2}{3}} \operatorname{sign}\left(\sigma_{0}\right)-K_{0} \sigma_{0}+\sigma_{1}
$$

where $\sigma_{1}=z_{1}-\dot{f}$.

Since $\hat{\lambda}_{0} \geq 0$ and $K_{0}>0$, from (33) we have:

$$
\sigma_{1}-\dot{\sigma}_{0}=\operatorname{sign}\left(\sigma_{0}\right)\left[\hat{\lambda}_{0}\left|\sigma_{0}\right|^{\frac{2}{3}}+K_{0}\left|\sigma_{0}\right|\right]
$$

and we can conclude that:

$$
\operatorname{sign}\left(\sigma_{1}-\dot{\sigma}_{0}\right)=\operatorname{sign}\left(\sigma_{0}\right)
$$

Subtracting $\dot{f}$ on both sides of the second equation of (29), then we obtain:

$$
v_{0}-\dot{f}=-\hat{\lambda}_{0}\left|\sigma_{0}\right|^{\frac{2}{3}} \operatorname{sign}\left(\sigma_{0}\right)-K_{0} \sigma_{0}+z_{1}-\dot{f}
$$

Substituting $\dot{f}$ by its expression (32) in (35), we have:

$$
\dot{\sigma}_{0}=-\tilde{\lambda}_{0}\left|\sigma_{0}\right|^{\frac{2}{3}} \operatorname{sign}\left(\sigma_{0}\right)-K_{0} \sigma_{0}
$$

with $\tilde{\lambda}_{0}=\hat{\lambda}_{0}-\lambda_{0}^{*}$, which is an error between the dynamic value of the gain and a priori one.

Considering now $\sigma_{1}=z_{1}-\dot{f}$, it gives $s_{1}=\sigma_{1}-\dot{\sigma}_{0}$. By subtracting $\ddot{f}$ from both sides of the last equation of (29) and taking into account the new expression of $s_{1}$, we have:

$$
\begin{aligned}
\dot{\sigma}_{1}= & -\tilde{\lambda}_{1}\left|\sigma_{1}-\dot{\sigma}_{0}\right|^{\frac{1}{2}} \operatorname{sign}\left(\sigma_{1}-\dot{\sigma}_{0}\right)-K_{1}\left(\sigma_{1}-\dot{\sigma}_{0}\right) \\
& -\tilde{\lambda}_{2} \int_{0}^{t} \operatorname{sign}\left(\sigma_{1}-\dot{\sigma}_{0}\right) d t
\end{aligned}
$$

Let us define a Lyapunov function as:

$V_{12}\left(\sigma_{0}, \sigma_{1}, \tilde{\lambda}_{i}\right)=\frac{1}{2} \sigma_{0}^{2}+\frac{1}{2}\left(\sigma_{1}-\dot{\sigma}_{0}\right)^{2}+\frac{1}{2} \sum_{i=0}^{2} \tilde{\lambda}_{i}^{2}, i \in\{0,1,2\}$

The equilibrium point is defined by $X_{e}^{\sigma, \tilde{\lambda}}=(0,0,0)$.

The derivative of this Lyapunov function is given by:

$$
\dot{V}_{12}=\sigma_{0} \dot{\sigma}_{0}+\left(\sigma_{1}-\dot{\sigma}_{0}\right)\left(\dot{\sigma}_{1}-\ddot{\sigma}_{0}\right)+\tilde{\lambda}_{0} \dot{\hat{\lambda}}_{0}+\tilde{\lambda}_{1} \dot{\hat{\lambda}}_{1}+\tilde{\lambda}_{2} \dot{\hat{\lambda}}_{2}
$$

then

$$
\sigma_{0} \dot{\sigma}_{0}+\tilde{\lambda}_{0} \dot{\hat{\lambda}}_{0}=-K_{0} \sigma_{0}^{2}
$$

and

$$
\left(\sigma_{1}-\dot{\sigma}_{0}\right) \dot{\sigma}_{1}+\tilde{\lambda}_{1} \dot{\hat{\lambda}}_{1}+\tilde{\lambda}_{2} \dot{\hat{\lambda}}_{2}=-K_{1}\left(\sigma_{1}-\dot{\sigma}_{0}\right)^{2}
$$

Substituting (40) and (41) in (39):

$$
\left.\dot{V}_{12}=-K_{0} \sigma_{0}^{2}-K_{1}\left(\sigma_{1}-\dot{\sigma}_{0}\right)^{2}-\left(\sigma_{1}-\dot{\sigma}_{0}\right) \ddot{\sigma}_{0}\right)
$$

We have

$$
\begin{aligned}
-\left(\sigma_{1}-\dot{\sigma}_{0}\right) \ddot{\sigma}_{0}= & -\left(\sigma_{1}-\dot{\sigma}_{0}\right)\left[-\dot{\hat{\lambda}}_{0}\left|\sigma_{0}\right|^{\frac{2}{3}} \operatorname{sign}\left(\sigma_{0}\right)-K_{0} \dot{\sigma}_{0}\right. \\
& \left.-\frac{2}{3} \tilde{\lambda}_{0}\left|\sigma_{0}\right|^{-\frac{1}{3}} \dot{\sigma}_{0}\right]
\end{aligned}
$$

By introducing the dynamic gain $\hat{\lambda}_{0}$ (see system (31)) in (43), the following equality is satisfied:

$$
\begin{aligned}
-\left(\sigma_{1}-\dot{\sigma}_{0}\right) \ddot{\sigma}_{0}= & -\mid \sigma_{1}-\dot{\sigma}_{0}\left[-\left|\sigma_{0}\right|^{\frac{7}{3}}+K_{0}^{2}\left|\sigma_{0}\right|\right. \\
& \left.+\frac{2}{3} \tilde{\lambda}_{0}^{2}\left|\sigma_{0}\right|^{\frac{1}{3}}+\frac{5}{3} \tilde{\lambda}_{0} K_{0}\left|\sigma_{0}\right|^{\frac{2}{3}}\right]
\end{aligned}
$$

Consequently, the equation (39) can be rewritten as follows:

$$
\begin{aligned}
\dot{V}_{12}= & -K_{0} \sigma_{0}^{2}-K_{1}\left(\sigma_{1}-\dot{\sigma}_{0}\right)^{2}-\frac{2}{3}\left|\sigma_{1}-\dot{\sigma}_{0}\right| \tilde{\lambda}_{0}^{2}\left|\sigma_{0}\right|^{\frac{1}{3}} \\
& -\left|\sigma_{1}-\dot{\sigma}_{0}\right|\left[-\left|\sigma_{0}\right|^{\frac{7}{3}}+\frac{5}{3} \tilde{\lambda}_{0} K_{0}\left|\sigma_{0}\right|^{\frac{2}{3}}+K_{0}^{2}\left|\sigma_{0}\right|(45)\right.
\end{aligned}
$$

To show that $\dot{V}_{12}$ is negative, it is sufficient to prove that :

$$
\Upsilon=\left[-\left|\sigma_{0}\right|^{\frac{7}{3}}+\frac{5}{3} \tilde{\lambda}_{0} K_{0}\left|\sigma_{0}\right|^{\frac{2}{3}}+K_{0}^{2}\left|\sigma_{0}\right|\right] \geq 0
$$

Therefore, let us assume that $\left|\tilde{\lambda}_{0}\right| \leq \tilde{\lambda}_{0 M}$, where $\tilde{\lambda}_{0 M}$ is a positive constant satisfying the following inequality:

$$
\tilde{\lambda}_{0 M}<\frac{3}{5} K_{0}\left|\sigma_{0}\right|^{\frac{1}{3}}
$$


In order to obtain the condition defined by (46), one must choose $K_{0}$ such that:

$$
K_{0}\left[\tilde{\lambda}_{0}+\frac{3}{5} K_{0}\left|\sigma_{0}\right|^{\frac{1}{3}}\right] \geq \frac{3}{5}\left|\sigma_{0}\right|^{\frac{5}{3}}
$$

It is obvious that it is always possible to find some value of $K_{0}$ (namely a high-value) satisfying both inequalities (47) and (48).

Under these conditions, we can conclude that $\dot{V}$ is a negative function $\forall\left(\sigma_{0},\left(\sigma_{1}-\dot{\sigma}_{0}\right), \tilde{\lambda}_{i}\right) \in \mathbb{R}^{3}$ but this property vanishes for $\left(0,0, \tilde{\lambda}_{i}\right)^{T} \neq X_{e}^{\sigma, \tilde{\lambda}}$. Consequently $\dot{V}$ is a globally seminegative definite function on $\mathbb{R}^{3}$ and it is a locally negative definite function on $\mathbb{R}^{3} \mid\left(0,0, \tilde{\lambda}_{i}\right)^{T}$. Therefore, with this Lyapunov function, a global convergence on $\mathbb{R}^{3}$ of the equilibrium point is proven. This means also that a local asymptotic convergence of the algorithm is only proven on $\mathbb{R}^{3} \mid\left(0,0, \tilde{\lambda}_{i}\right)^{T}$. It is possible here to use the LaSalle's invariance principle, but precisely in our case, it does not allow to conclude on the global asymptotic convergence of system equilibrium point.

Thereby, the given proof insures just the convergence of the estimation errors $s_{0}$ and $s_{1}$ to zero, but the convergence of dynamic gains to $\lambda_{0}^{*}$ and $\lambda_{1}^{*}$ is not guaranteed. Moreover, these dynamic gains change over time according to the imposed dynamic laws, which have a bounded evolution depending on the initial values of the algorithm gains. Obviously, any dynamic algorithm is sensitive to its initial values. In our case, the influence of the initial values on the convergence algorithm has not still been theoretically studied. However, several simulation tests were performed with different initial values and without any convergence problems.

\section{Simulation Results}

In order to examine the performances of the proposed algorithms over the classic one, a comparative study is proposed in this section. Many criteria are considered. The first one is the accuracy of the algorithms with respect to the magnitude and the phase shift due to the estimation of derivative signal. The second one involves studying the robustness of the different algorithms against noise. In our context, the robustness is defined by the reduction of the noise amplification rate on the output signal. To simplify the figures titles, the following abbreviation are used: Super Twisting: ST, 2nd-Order Differentiator: 2OD, New scheme for 1st-Order Differentiator: N1OD, New scheme for 2nd-Order Differentiator: N2OD.

\section{A. Simulation Tests Without Noise}

Let us consider a noiseless input signal defined by $h(t)=$ $\cos (0.6 \pi t)$. Since the input signal is already known and not corrupted by any noises, its Lipschitz constant is also known. Thus, with using the Levant condition (3), the gains of the classic algorithms (ST, 2OD) are properly adjusted. For the proposed algorithms, the initial values of the dynamic gains are chosen equal to zero and its convergence gains are selected such that: N1OD $\left(K_{0}=500\right)$ and N2OD $\left(K_{0}=500, K_{1}=\right.$ 800). To compare the obtained results, the absolute value of the maximum error $\left|e_{\max }\right|$ is regarded as one of the comparison criteria. This error is the difference between the estimate of the $i^{t h}$ derivative and the analytical derivative signal. Other criterion is also taken into account which is the maximum phase-shift $\left|\Delta \varphi_{i}\right|_{\max }\left(^{\circ}\right)$ resulting by the estimate of the $i^{t h}$ derivative. In order to estimate the 2nd-derivative of the input signal using ST and N1OD, which are a 1st-order differentiator, two blocks of these algorithms are arranged in cascade for acting as a 2nd-order one.

TABLE I: Estimation error and phase-shift

\begin{tabular}{|c|c|c|c|c|}
\hline Algorithm & ST & N1OD & 2OD & N2OD: \\
\hline$\left|e_{\max 1}\right|$ & 0.024 & 0.0017 & 0.00019 & 0.00018 \\
\hline$\left|e_{\max 2}\right|$ & 0.196 & 0.06 & 0.016 & 0.011 \\
\hline$\left|\Delta \varphi_{1}\right|_{\max }\left(^{\circ}\right)$ & 0.047 & 0.046 & 0.045 & 0.045 \\
\hline$\left|\Delta \varphi_{2}\right|_{\max }\left(^{\circ}\right)$ & 0.07 & 0.048 & 0.047 & 0.05 \\
\hline
\end{tabular}

The table I shows that with a higher order sliding modes differentiators we can expect a low error with a small phaseshift. This is can be explained by the properly tuning of the algorithms gains. Regarding to the magnitude error, the $\mathrm{DO} 2$ and the N2OD present relatively similar values. However, the estimated signals (first and second order) given by the N1OD are more accurate than obtained by ST. In fact, the error values are improved by a factor over to 14 for the 1st-order estimate and by a factor of 3 for the 2nd-order estimate.
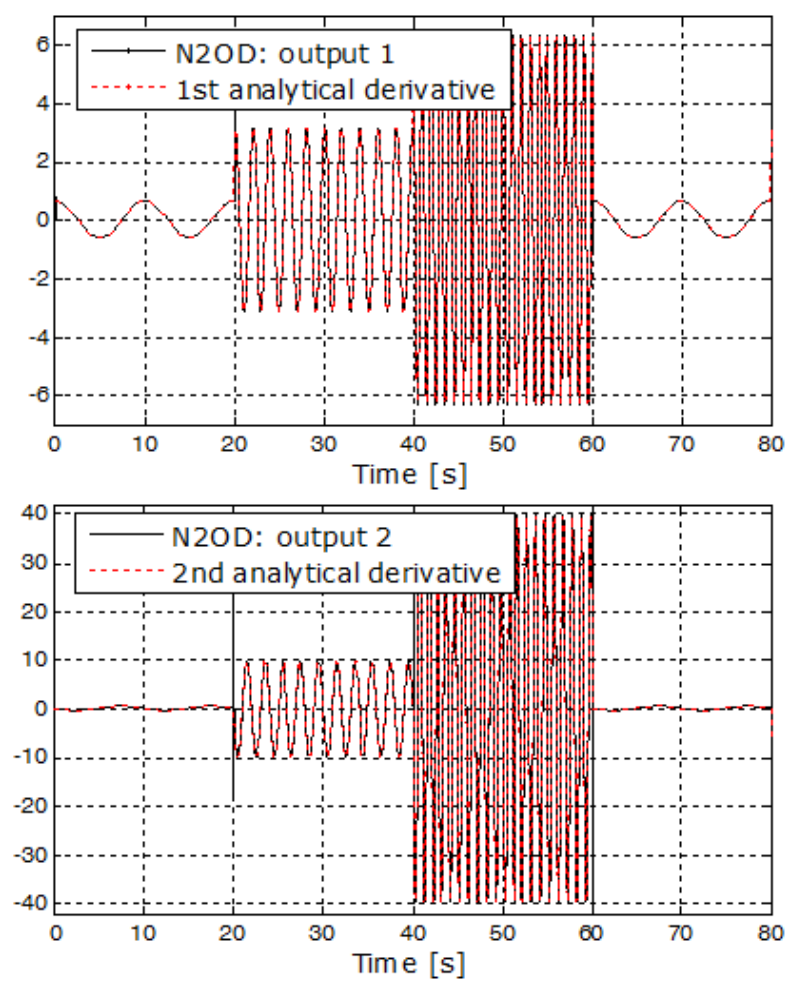

Fig. 2: N2OD Outputs: variable frequency signal

Another test simulation is done in order to show the behaviour of the proposed algorithm for a multi-frequency signal. For 
this test, only the 2nd-order algorithm results are provided. The setting of the gains algorithm are given such as $K_{0}=80$, $K_{1}=70, \hat{\lambda}_{0}(0)=0, \hat{\lambda}_{2}(0)=0$ and $\hat{\lambda}_{2}(0)=0$. The chosen signal is give by: $h_{1}(t)=\sin \left(2 f_{1} \pi t\right)$, where the frequency $f_{1}$ varies according to the following sequence $(0.1 \mathrm{~Hz}, 0.5 \mathrm{~Hz}, 1 \mathrm{~Hz})$. From figure 2, the estimated signals are too close to the analytical one despite input signal frequency variation.

\section{B. Simulation Tests With Noise}

In this section, the differentiation tests of noisy input signal are handled. So to show the behaviour of the studied and proposed differentiator for such signal, a white Gaussian noise with zero mean and a standard deviation equal to 0.03 is added to the basic signal $h(t)$ that is used in the previous section.

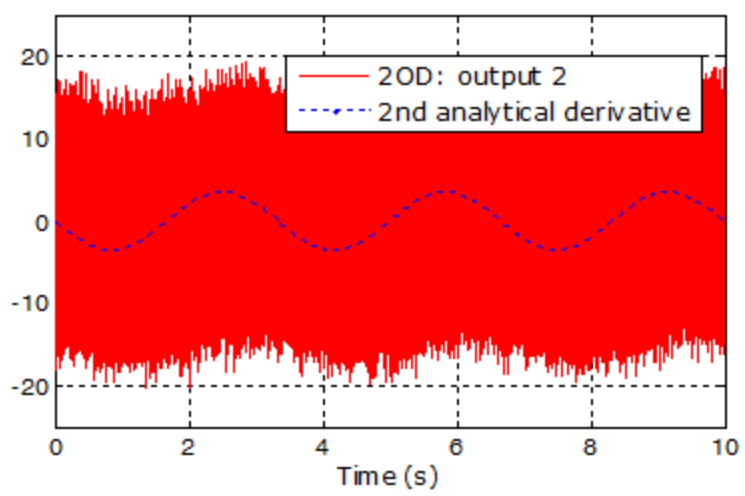

(a) $2 \mathrm{OD}\left(\lambda_{0}=8, \lambda_{1}=7, \lambda_{1}=3\right)$

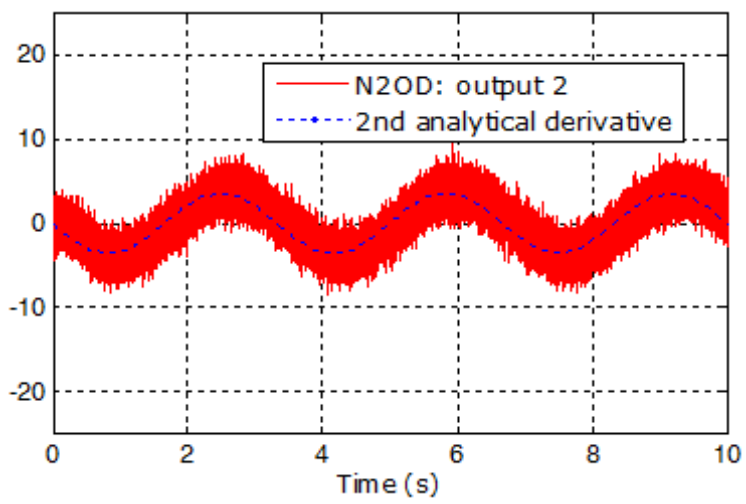

(b) $\mathrm{N} 2 \mathrm{OD}\left(K_{0}=7, K_{1}=4\right)$

Fig. 3: Estimation of the 2nd-derivative

From figures (3) and (4), we emphasize that the estimate of the 1st-derivative is more smooth than the second one. In spite of the similar setting gains (convergence gains for the new schemes) of the two schemes, it is worth noting that the results provided by the proposed algorithms have significantly reduced noise amplification compared to the classical one. This is illustrated by figure (3) where the noise amplification is 7 times lower using N2OD than the noise level given by 2OD. These results are mainly owned to the additional linear component, which is the continuous one, into the equations of the new schemes.

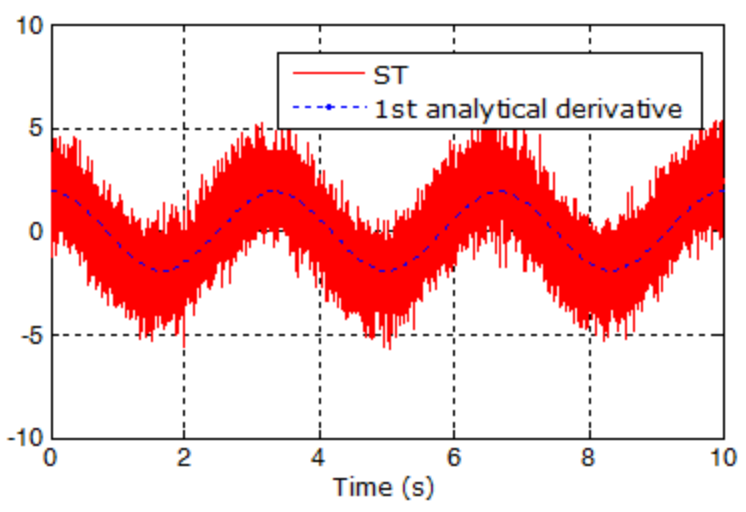

(a) $\operatorname{ST}\left(\lambda_{0}=10, \lambda_{1}=8\right)$

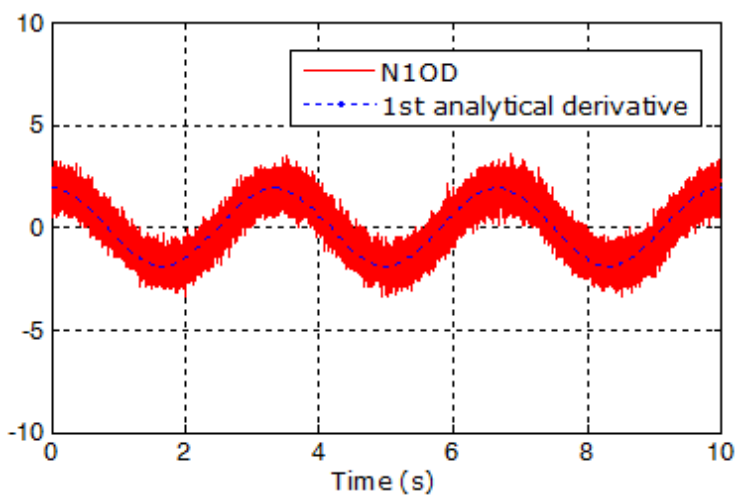

(b) N1OD $\left(K_{0}=8\right)$

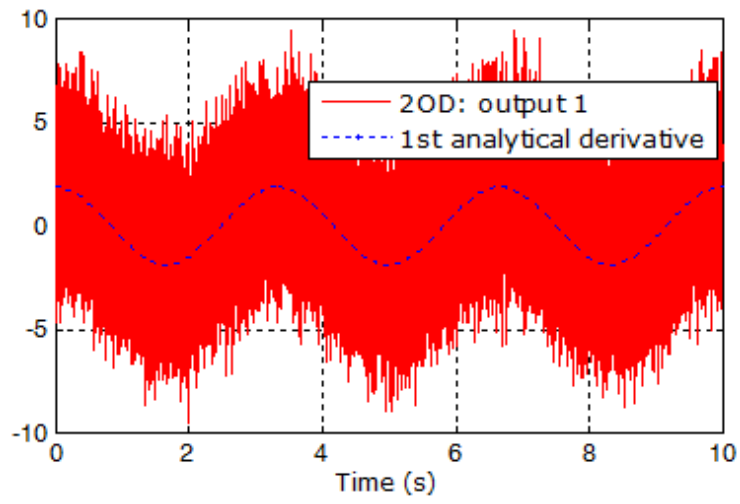

(c) $2 \mathrm{OD}\left(\lambda_{0}=8, \lambda_{1}=7, \lambda_{1}=3\right)$

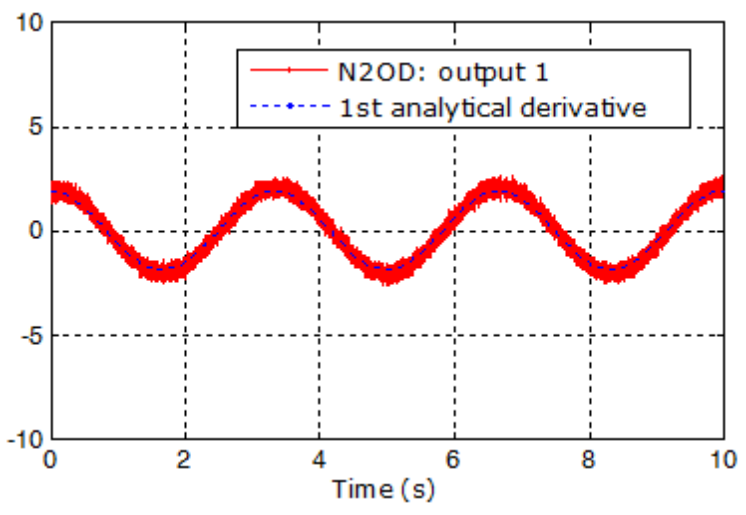

(d) $\mathrm{N} 2 \mathrm{OD}\left(K_{0}=7, K_{1}=4\right)$

Fig. 4: Estimation of the 1st-derivative 
So it is interesting to note that one of the major contributions of these proposed differentiators is the notable decrease of the noise amplification on the estimating derivatives. Since the measurement noise is inevitable in practice, this advantage may have a strong interest.

\section{Remarks about the setting of the proposed algorithms:}

With noiseless signals, if the chosen of the convergence gains values $K_{i}, i \in\{1,2\}$ becomes high then the convergence time of the algorithms becomes quick. However, with noisy signals, there are some compromise between the reduction of noise amplification and the convergence time of the differentiators. Indeed, the smoothing of the outputs differentiators is due to the presence of the linear terms $K_{i} s_{i}$. Then, it is necessary not to choose too high values of $K_{i}$ in this case. It should also be noted that it is necessary to impose lower initial values of the dynamic gains to reduce as much as possible the effect of the discontinuous components, so to reduce the chattering effect. Other remark according to the dynamic gains $\hat{\lambda}_{0}$ and $\hat{\lambda}_{1}$, it is necessary to reset their values after some computation time otherwise these values becomes so high.

In the next sections, the goal is to investigate the efficiency of such algorithms into the control-loop of an electro-hydraulic system where its model is described in the following section.

\section{ELECTRO-HYDRAULIC SYSTEM MODEL}

\section{A. Presentation of the test bench}

The test bench (see figure 5) is a symmetric double acting electro-hydraulic servo-drive consisting in double rod cylinder controlled by two five-way servovalves. The characteristics of the hydraulic actuator are given in the table II (see appendix). The servovalves are electro-hydraulic servomechanisms that constitute the main interface between the electrical control signal and the hydraulic actuator. These modulators, developed by MOOG, can provide a quite large bandwidth, thus a short response time, and a good precision. As indicated in the MOOG data sheet, the bandwidth reaches up to $1 \mathrm{kHz}$ for $5 \%$ of spool displacement and a rated flow up to $19(\mathrm{l} / \mathrm{min})$ at 70 bar pressure drop at full opening.

A perforated block is designed specifically for this test bench. It allows the implementation of the two servovalves and of other components that enable different operating modes for the system. Thus the actuator chambers can be fed either by a $5 / 2$ single servovalve or by two servovalves in parallel to increase the flow. The servovalves can also be used in three-way mode to supply flow independently to each actuator chamber. To reach this modularity, the perforated block is equipped with two solenoid valves and two flow regulators. Spherical accumulators are also mounted on this block to avoid pressure peaks. However, this block introduces parasite phenomena (pressure drop, capacitive and inertial effects) that could present a significant influence on the overall behaviour of the system in some circumstances. The simplified diagram of the servo-system is given in figure 6 .
In this paper, a single mode is considered to control the actuator by one $5 / 2$ servovalve.

\section{B. Model system}

The model used for the control relies on several assumptions. The first one consists to only take into account the resistive effect produced by the perforated block. This hypothesis will be later justified by the choice of the trajectory.

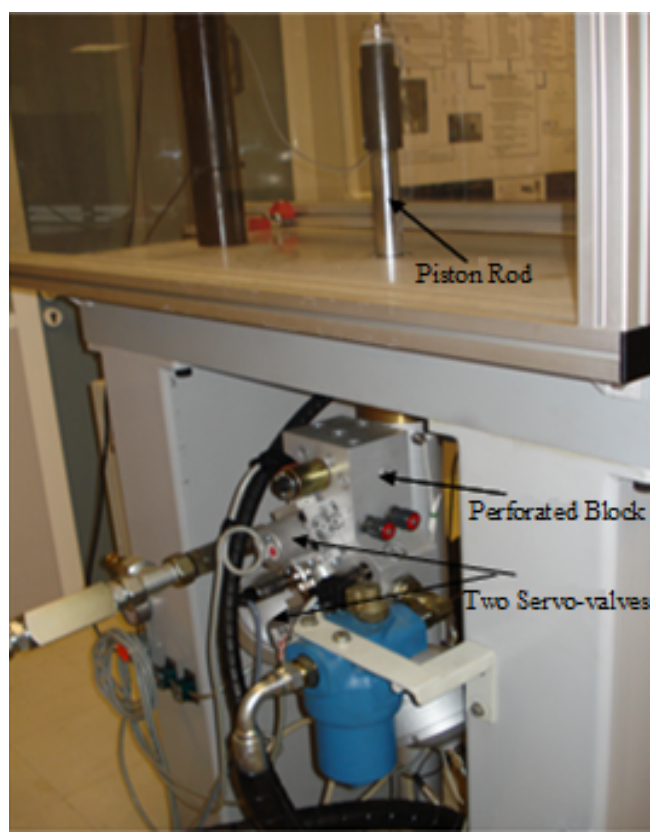

Fig. 5: Electro-hydraulic test bench

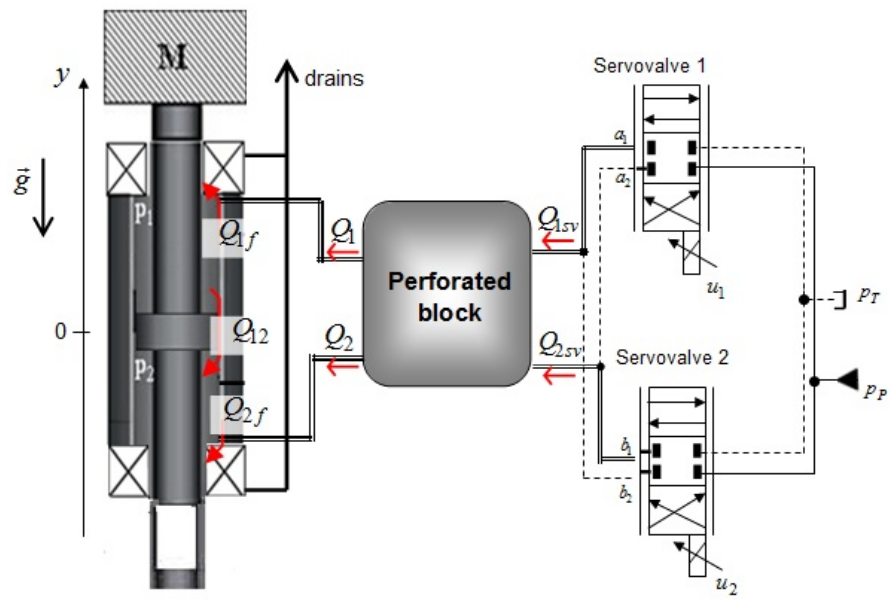

Fig. 6: Simplified diagram of servo-system

According to the second law of Newton, the load mechanical equation is given by:

$$
M a=S \Delta P-M g-b v+h_{2}(v(t))
$$

where $M$ is the mass of the moving part, $\Delta P=p_{1}-p_{2}$ is the pressure drop across the load piston, $S$ is the effective area of the actuator chambers, $b$ represents the coefficient of 
the viscous friction force, $v, a$ are respectively the velocity and the acceleration of the load. Dry friction forces depend explicitly on velocity and is represented by the function $h_{2}(v(t))$.

Friction is a common phenomena for all mechanical systems and its effects have been widely studied [18]. Its influence is mostly noticeable at low velocities on account of a high gain variation around a null velocity. This leads to achieve an accurate model which requires refinement of the analytical description of these phenomena. In tribology, many friction models have been proposed. These models can be differentiated with respect to their degree of complexity which is equivalent to the phenomena that is taken into account during the modeling step. For example, the well-known quasi-static friction model, known as Tustin model, considers Coulomb friction, viscous friction and Stribeck effect, [19]. In [18], a dynamic model of friction enables the "stick/slip" phenomena to be described by considering the materials properties. Aside the nonlinearity of these models, they usually have a discontinuity around null velocity. Therefore, in many applications the control law is synthesized with disregarding dry friction as it is done in [20]. This hypothesis is always made for the synthesis of backstepping control law because of such controller requires the derivative of friction.

In our work, the dry friction has been identified at low velocities from experimental trials using equation (49). The results are obtained for several cycles around the same operating point and in the same experimental conditions. Different phases of friction can be observed. With the hydrodynamic bearings technology used in our actuator, the slope is not too stiff around null velocity. In fact, such bearing technology allows the formation of an oily film, even at very low velocity. This explains that the static dry friction magnitude is small compared to what is observed in conventional actuators.

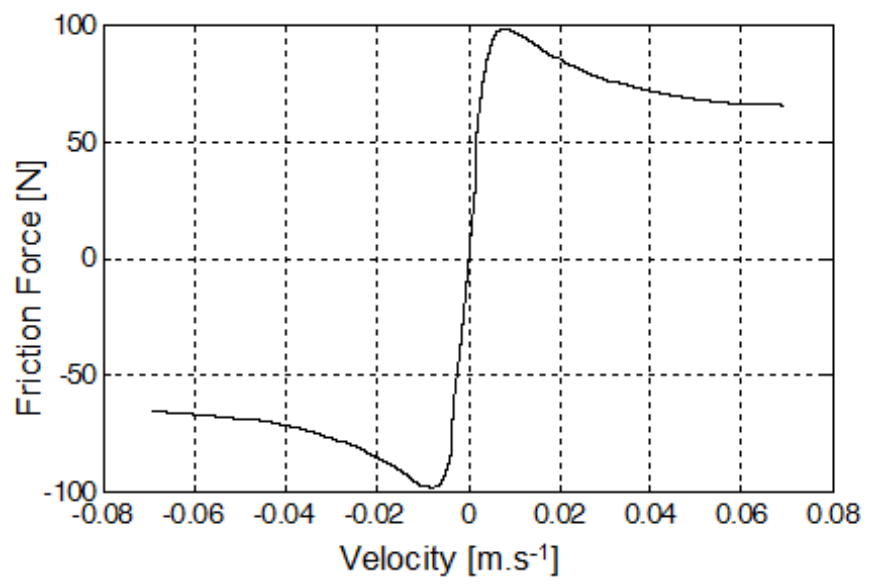

Fig. 7: Simulation model of friction

In our case, the dry friction can be approximated around null velocity by a smooth function such as a $\tanh ($.$) instead of the$ $\operatorname{sign}($.$) function which is a discontinuous one. Then, the fric-$ tion can be defined by a nonlinear and a differentiable function $h_{2}(v(t))$. By repeating several times the same experimental test, we obtained an average maximum value of the friction about $\pm 150 \mathrm{~N}$ for a supply pressure $p_{P}=210 \mathrm{bar}$. This corresponds to only $0.75 \%$ of the maximum force that can be developed by the cylinder $\left(20 \mathrm{kN}\right.$ for $p_{P}=210$ bar $)$. A nonlinear model based on the well-known Tustin friction model [19] was proposed assuming that the dry friction is symmetric according to velocity and the hysteresis effect is negligible:

$$
h_{2}(v(t))=\left[F_{\text {sdyn }}+\left(F_{\text {sdyn }}-F_{C}\right) e^{-C_{1}|v|}\right] \tanh \left(\frac{v(t)}{v_{0}}\right)
$$

Where $F_{s d y n}$ is the dynamic dry friction, $F_{C}$ is the Coulomb friction. $C_{1}$ and $v_{0}$ are respectively the coefficient of Stribeck effect and the constant value for velocity scaling.

Using the above assumptions, the empirical model of the friction is then reduced to the simluation model for low velocities given by figure 7 .

The flow balance in a variable volume chamber is obtained assuming that: i) both the temperature and the pressure of the oil are homogeneous in each chamber. ii) the oil density variation is small compared to its average density, iii) the temperature variation is small compared to the average temperature which is equal to the oil supply temperature.

Using the definition of the isothermal bulk modulus which is inverse of the oil compressibility at a given temperature, the nonlinear equations that describe the fluid flow distribution in the servovalve can be written in their simplest forms, [1]:

$$
\left\{\begin{array}{l}
Q_{1}-Q_{\text {leakage }}=\frac{V_{1}(y)}{\beta} \frac{d p_{1}}{d t}+\frac{d V_{1}}{d t} \\
Q_{\text {leakage }}-Q_{2}=\frac{V_{2}(y)}{\beta} \frac{d p_{2}}{d t}+\frac{d V_{2}}{d t}
\end{array}\right.
$$

According to the manufacturer data, the inter-chamber leakage flow of is equal to $Q_{\text {leakage }}=10^{-4}(\mathrm{l} / \mathrm{min})$ at a $\Delta P=70$ bar and therefore this flow rate can be disregarded in equation (51). $V_{1}$ and $V_{2}$ are the total volumes of the cylinder defined respectively by:

$$
\left\{\begin{array}{l}
V_{1}(y)=V_{01}+S y \\
V_{2}(y)=V_{02}-S y
\end{array}\right.
$$

where $V_{01}=V_{M 1}+S \frac{l}{2}$ and $V_{02}=V_{M 2}+S \frac{l}{2}$ are the volumes of the chambers at piston central position, $y$ is the displacement of the load, $l$ is the cylinder stroke and $V_{M 1}$, $V_{M 2}$ are both sides dead volumes of the cylinder. The piping volumes of the perforated block are also taken account in $V_{M 1}$ and $V_{M 2}$.

The two servovalves are considered identical, symmetric and controlled by the same input signal $u$. These power modulators present a large bandwidth compared to the actuator dynamic, so we can assumed that the spool valve displacement $x_{t}$ is directly related to the control voltage $u$ and given by $x_{t}=$ $K_{s v} u$. Let us note that this assumption is only valid in some operating mode [21], considering the flow regime as mainly turbulent, the flow rate (51) can be written in affine form with 
a single input:

$$
\left\{\begin{array}{l}
Q_{1 s v}=Q_{1}=\alpha u C_{1}^{*} \psi_{1}\left(p_{1}, p_{P}, p_{T}, \operatorname{sign}(u)\right) \\
Q_{2 s v}=Q_{2}=\alpha u C_{2}^{*} \psi_{1}\left(p_{2}, p_{P}, p_{T}, \operatorname{sign}(u)\right)
\end{array}\right.
$$

with $\alpha=K_{t} K_{s v} \sqrt{\frac{2}{\rho}} C_{d}$ is assumed to be a constant parameter where $\rho$ is the fluid density and $C_{d}$ the flow coefficient. Thus, equation (53) can be written as :

$\left\{\begin{array}{l}\psi_{1}\left(p_{1}, p_{P}, p_{T}, \operatorname{sign}(u)\right)=\left[h_{3}(u) G_{11}(.)+h_{3}(-u) G_{12}(.)\right] \\ \psi_{1}\left(p_{2}, p_{P}, p_{T}, \operatorname{sign}(u)\right)=\left[h_{3}(u) G_{21}(.)+h_{3}(-u) G_{22}(.)\right]\end{array}\right.$

with

$$
\left\{\begin{array}{l}
G_{11}(.)=\sqrt{\left|p_{P}-p_{1}\right|} \operatorname{sign}\left(p_{P}-p_{1}\right) \\
G_{12}(.)=\sqrt{\left|p_{1}-p_{T}\right|} \operatorname{sign}\left(p_{1}-p_{T}\right) \\
G_{21}(.)=\sqrt{\left|p_{2}-p_{T}\right|} \operatorname{sign}\left(p_{2}-p_{T}\right) \\
G_{22}(.)=\sqrt{\left|p_{P}-p_{2}\right|} \operatorname{sign}\left(p_{P}-p_{2}\right)
\end{array}\right.
$$

and $h_{3}(u)=\frac{1+\operatorname{sign}(u)}{2}$. Due to the asymmetry of the circuit in this operating mode, the constants $C_{1}^{*}, C_{2}^{*}$ have a different values. These values were determined following specific tests in order to consider the pressure drop into the perforated block.

Each state variable is physically bounded. Thus the physical domain of the system can be described by:

$$
D_{\phi}=\left\{(y, v, a) \in \mathrm{R}^{3} /|y| \leq \frac{l}{2}, p_{1}, p_{1} \in \Gamma_{p} \equiv\right] p_{T}, p_{P}[\}
$$

Using (56), the terms $\operatorname{sign}\left(p_{P}-p_{j}\right)$ and $\operatorname{sign}\left(p_{j}-p_{T}\right)$, with $j=\{1,2\}$ introduced into (55) can be eliminated, as well as the absolute values.

The system model can then be put in the normal form as shown below:

$$
\dot{X}=f_{1}(X)+g_{1}(X) u,
$$

with $X, f_{1}(X), g_{1}(X) \in \mathrm{R}^{4}, u \in \mathrm{R}$ and $X=\left[y, v, a, p_{1}\right]^{T}$. $f_{1}$ and $g_{1}$ are vector fields locally Lipschitz, defined by:

$$
\begin{aligned}
& f_{1}(X)=\left(\begin{array}{c}
v \\
\frac{1}{M}\left[S\left(p_{1}-p_{2}\right)-g-b v-h_{2}(v(t))\right] \\
-S \frac{\beta}{V_{1}(y)} v \\
S \frac{\beta}{V_{2}(y)} v
\end{array}\right)^{T} \\
& g_{1}(X)=\left(\begin{array}{c}
0 \\
0 \\
\frac{\beta}{V_{1}(y)} \alpha C_{1}^{*} \psi_{1}\left(p_{1}, p_{P}, p_{T}, \operatorname{sign}(u)\right) \\
-\frac{\beta}{V_{2}(y)} \alpha C_{2}^{*} \psi_{2}\left(p_{2}, p_{P}, p_{T}, \operatorname{sign}(u)\right)
\end{array}\right)
\end{aligned}
$$

\section{EFFectiveness Of Proposed DifFERENTIATOR Design In Control Purpose}

In this section, we address the problem of position trajectorytracking for the servo-system using a minimum number of mechanical sensors. A control law is synthesized in this paper based on the well-known backstepping technique. Such controller requires a position sensor, a velocity sensor and also an accelerometer. However, the accelerometer that is installed on the system has an accuracy of $\pm 2 \%$ of the operation range $( \pm 100) g$ which is very large according to the chosen position trajectories. Therefore, at low acceleration the output signal of this sensor is almost unusable for the closed-loop controller. Furthermore, no velocity sensor is set up on the test bench.

All these reasons could justify the benefit of using the real time numerical differentiators. Then, the aim of the experimental test is to study the influence of 2nd-order differentiator (2OD or N2OD) on the control law of the system. A comparative study between these two algorithm schemes (2OD and N2OD) is also performed.

Remark that the relative degree of the output system is equal to three compared to the system order which is equal to four. So the existence of 1 st-order residual dynamic in $p_{1}$ throughout $D_{\phi}$ is inevitable. With the backstepping controller, the asymptotic stability of the controlled part is insured. For the pressure residual dynamic, the asymptotic stability with meaning of the zeros dynamic [22] was already been demonstrated in [23].

The obtained control law is given as:

$$
\begin{aligned}
u= & \frac{1}{\frac{S \beta}{M}\left[\frac{\alpha C_{1}^{*} \psi_{1}(.)}{V_{1}(y)}+\frac{\alpha C_{2}^{*} \psi_{2}(.)}{V_{2}(y)}\right]}\left[-f_{1}(X)+\dot{a}_{d}-K_{11} e_{1}\right. \\
& \left.-K_{21} e_{2}-K_{31} e_{3}\right]
\end{aligned}
$$

with

$$
\left\{\begin{array}{l}
K_{11}=c_{1}^{3}-2 c_{1}-c_{2}, \quad c_{3}>0 \\
K_{21}=1-c_{1}\left(c_{1}+c_{2}\right)-c_{2}^{2} \\
K_{31}=c_{1}+c_{2}+c_{3}
\end{array}\right.
$$

and

$$
\left\{\begin{array}{l}
e_{1}=y-y_{d} \\
e_{2}=v-v_{d}+c_{1} e_{1}, \quad c_{1}>0 \\
e_{3}=a-a_{d}+e_{1}\left(c_{1}^{2}-1\right)-e_{2}\left(c_{1}+c_{2}\right), \quad c_{2}>0
\end{array}\right.
$$

$y_{d}, v_{d}$ and $a_{d}$ are the desired trajectories respectively of the position $y$, the velocity $v$ and the acceleration $a$. 
To show the performances of the differentiation algorithms, the velocity and the acceleration are reconstructed using a 2nd-order differentiators (2OD or N2OD), as shown in the diagram given below:

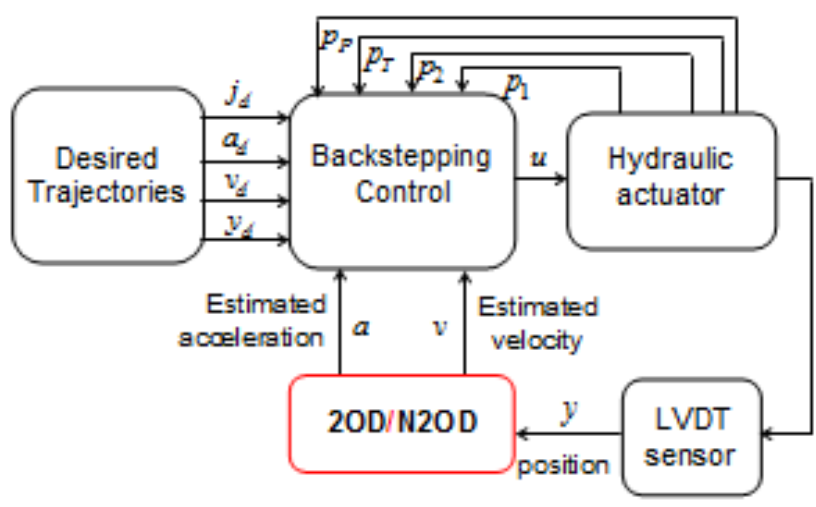

Fig. 8: Schematic diagram: "Controller/Differentiator"

Our approach of "Controller/Differentiator" is validated via Dspace 1104 controller board with a dedicated digital signal processor. The sampling frequency of the control loop is set to $1 \mathrm{kHz}$. As already noted, the relative degree of the position is equal to three. This means that the electro-hydraulic system can only track position trajectory at least three times differentiable. Moreover, to satisfy the assumptions made during the modeling phase, the chosen trajectories are settled on low and medium frequency.

In order to examine the performance of N2OD compared to 2OD, two tests have been applied. In the first one (test 1), a sinusoidal reference position is applied with $\pm 30 \mathrm{~mm}$ as a magnitude and $0.5 \mathrm{~Hz}$ as a signal frequency. For the second test (test 2), the same reference position is used but with different magnitude and frequency values which are chosen respectively as $\pm 50 \mathrm{~mm}$ and $1 \mathrm{~Hz}$.

The gains of the 2OD differentiator are set to $\lambda_{0}=18$, $\lambda_{1}=l 5, \lambda_{2}=8$ and those of N2OD are chosen so that $K_{0}=25, K_{1}=35$. For N2OD, the dynamic gains are initialized to zero. The choice of these gain values has been made in order to have a good precision and an acceptable quality of the estimates according to the input reference defined in test 1 .

The choice of the control law parameters is given such as $: c_{1}=400, c_{2}=400$ and $c_{3}=400$. The interest of the proposed experimental tests (test 1 and test 2) is that the parameters tuning of the "controller-differentiator" blocks remains unchanged during these experiments.

In order to compare the getting results; we make an analytical study based on multiple criteria which are made up of quantitative and qualitative criteria. The quantifiable criteria are chosen as follows: maximum position tracking error $e_{y_{\max }}$, maximum velocity error $e_{v \max }$ and maximum acceleration error $e_{a \max }$. As stated before, references (sensors) are not available on the test bench. Then, the computed errors are defined as the difference between the measured variable (position) or the estimated one (velocity, acceleration) and the corresponding desired trajectory. About the qualitative criterion, the quality of the estimated signal in terms of noise attenuation is taken into account.

\section{A. Test 1: experimental results}

Start us with the results obtained by 2OD. From the figure 9, the absolute value of the maximum position error is about 0.18 $\mathrm{mm}$. As we can see in the figure 12, the estimated of velocity and acceleration have practically no phase-shift compared to the desired signals. This good quality of the obtained signals is related to the good setting of the 2OD-gains with respect to the input signal relative to test 1 .

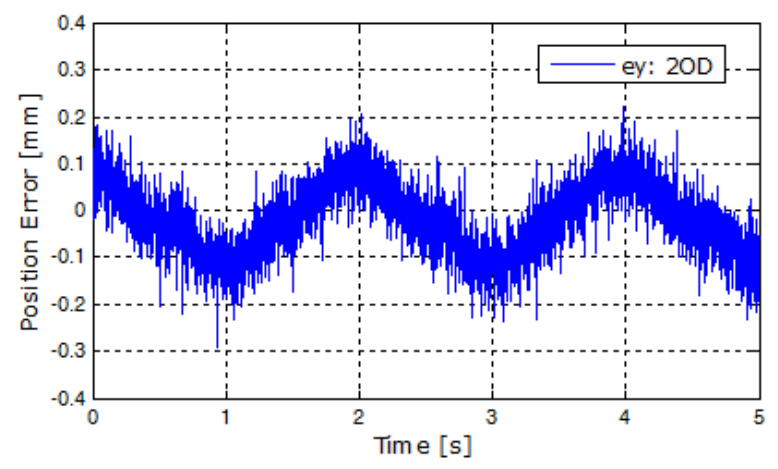

Fig. 9: Tracking position error [mm]: (2OD,test 1)

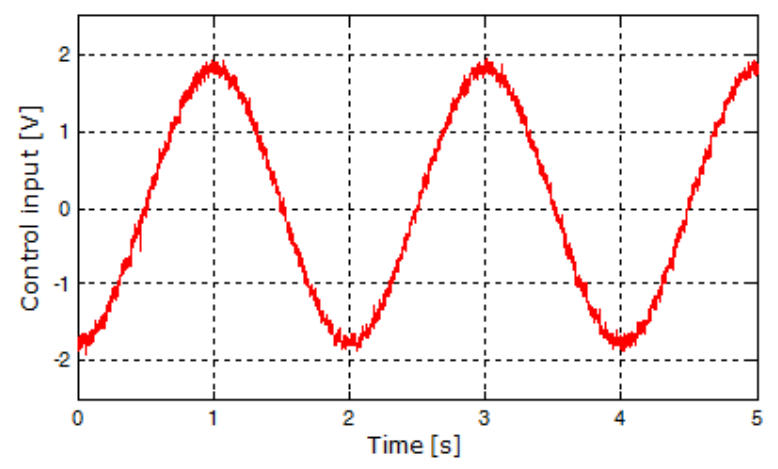

Fig. 10: Control input [V]: (2OD,test 1)

In spite of an appropriate choice of the gains values of 2OD, the estimated derivatives are relatively noisy. Figure 10 shows the obtained command signal for the "2OD-command" scheme. Figure 11 presents the position error using the N2OD algorithm. The maximum value (absolute value) is identical to the one obtained with the 2OD. The phase-shift is also very low. 


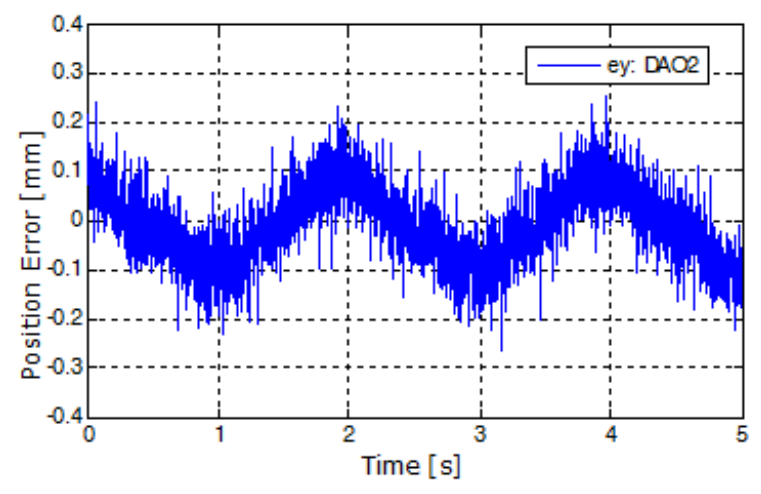

Fig. 11: Tracking position error [mm]: (N2OD,test 1)

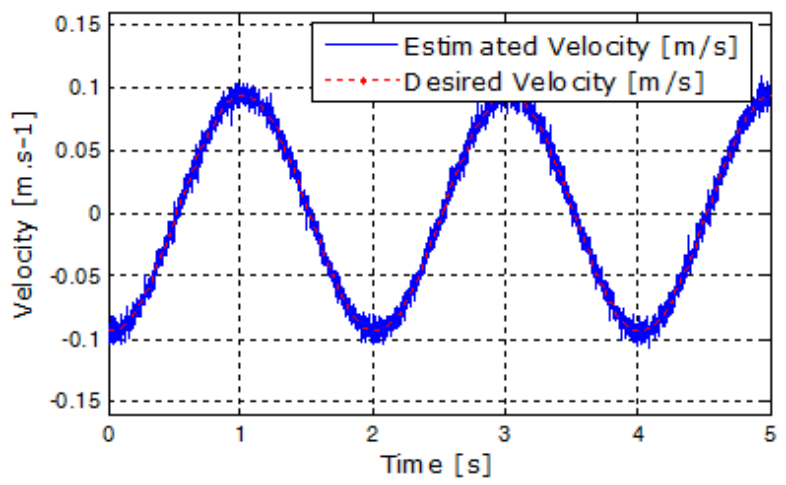

(a) Estimated velocity

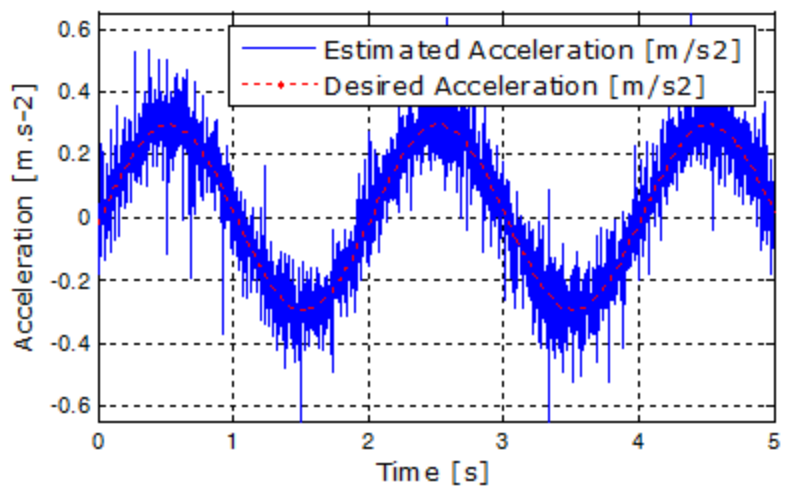

(b) Estimated acceleration

Fig. 12: Estimation velocity and acceleration: (2OD, test 1$)$

Regardless of differentiator (2OD/N2OD) used, there is no noticeable difference for both control signal and position error, so it is difficult to conclude of the effectiveness of the proposed algorithm according these both criteria. That is why; it is interesting to see the results which are given on the test 2 .

From the test 1 results, we can emphasize that the same maximum value in position error is provided in both cases (2OD and N2OD). However, the estimated signals by the $\mathrm{N} 2 \mathrm{OD}$ (figure 13) are less corrupted by noises than those estimated by the 2OD.

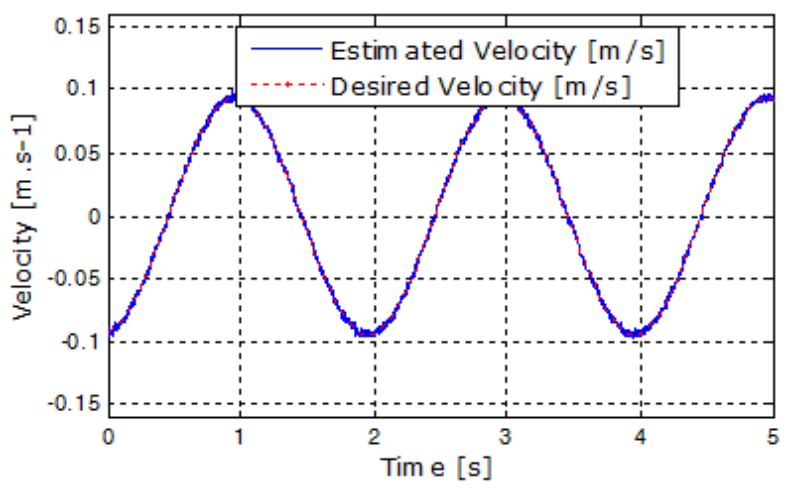

(a) Estimated velocity

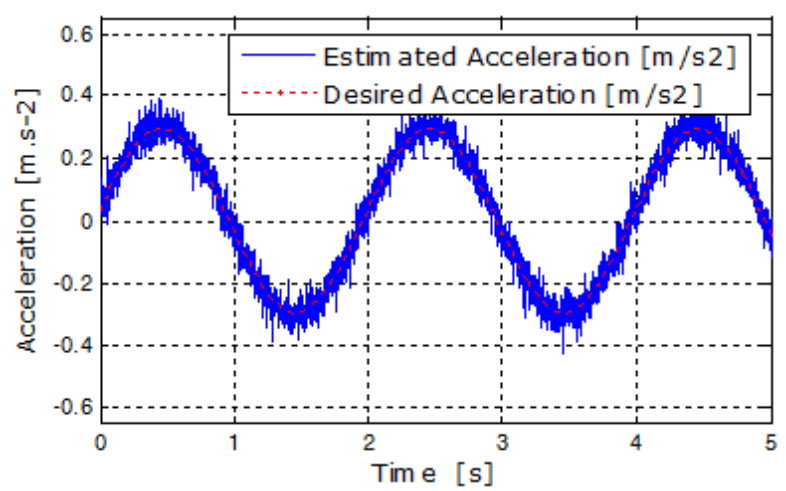

(b) Estimated acceleration

Fig. 13: Estimation velocity and acceleration: (N2OD, test 1)

\section{B. Test 2: experimental results}

The purpose of this second experimental test is to show more clearly the advantage provided by the new scheme of the 2nd-order differentiator. Recall that for this new trajectory, the parameters of the "controller-differentiator" blocks are unchanged and identical to those chosen for test 1 .

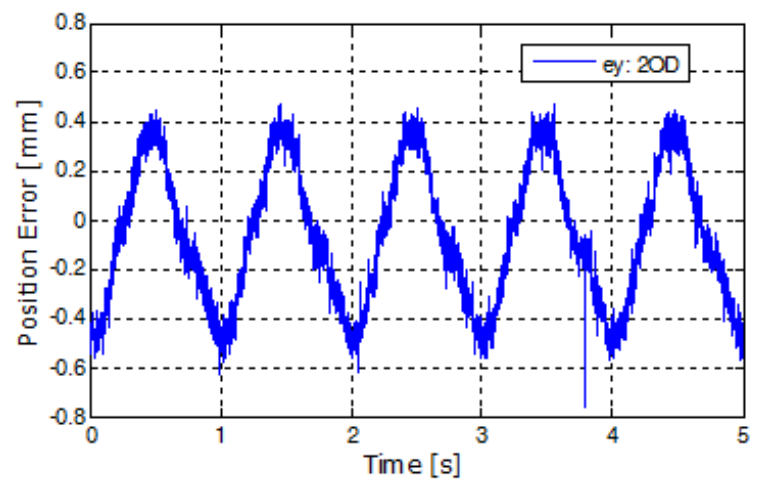

Fig. 14: Tracking position error [mm]: (2OD,test 2$)$

With using the 2OD, the maximum value of the position error is now about $0.5 \mathrm{~mm}$, (figure 14), which is increased compared to its value obtained in test 1 . This augmentation of the error can be explained by two reasons. The first one is the increase of the errors on the estimated signal. The second one is the change of the parameter uncertainties around the 
new operating range that is due to the change of the reference trajectory. On figure 15, the estimated acceleration shows a phase-shift which comes from a maximum acceleration error that equals to $0.64 \mathrm{~m} . \mathrm{s}^{-2}$. For the estimated velocity, the phase-shift is not noticeable but the maximum velocity error is equal to $0.011 \mathrm{~m} . \mathrm{s}^{-1}$.

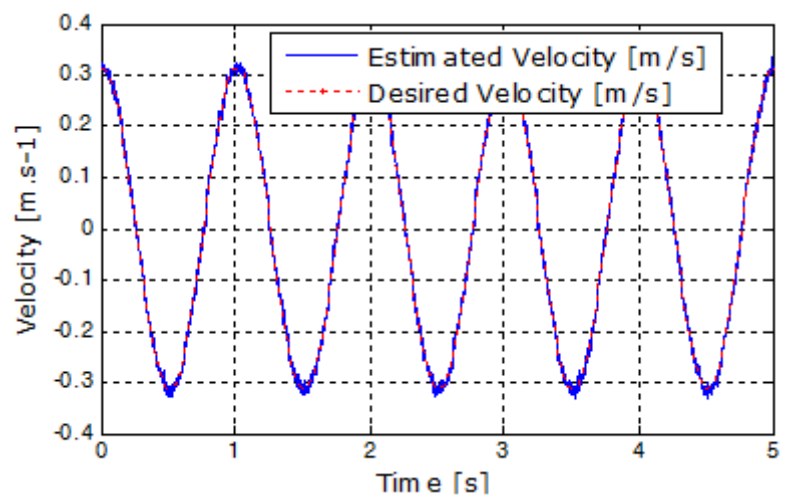

(a) Estimated velocity

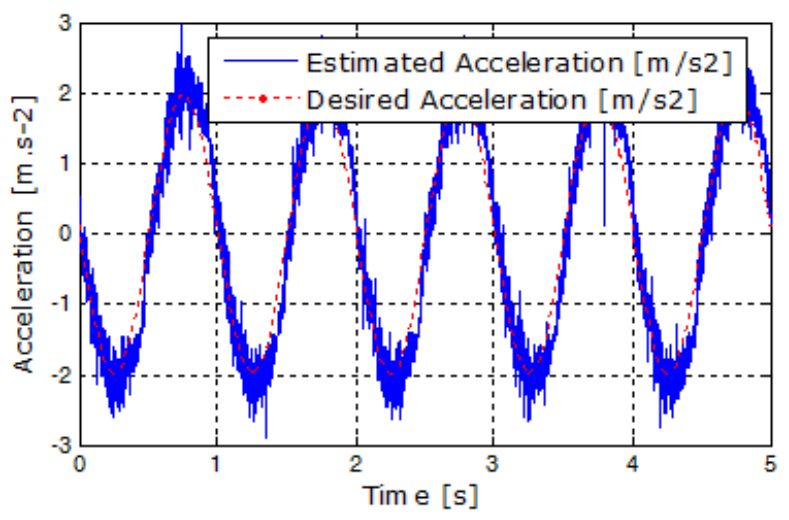

(b) Estimated acceleration

Fig. 15: Estimation velocity and acceleration: (2OD, test 2)

For N2OD, the position error and the control input are relatively similar than those given by 2OD. Consequently, only the curves of the estimated velocity and acceleration are presented. From figure 16, it is clear that the estimates given by the N2OD have a smaller phase-shifts and also better filtered than those given by the 2OD, especially for the acceleration signal. With the $\mathrm{N} 2 \mathrm{OD}$, the recorded maximal velocity error is approximately the same as the one given by the 2OD. However, the maximal error of acceleration is equal to $0.48 \mathrm{~m} . \mathrm{s}^{-2}$, which is significantly lower than the error given by the $2 \mathrm{OD}$.

Following these experimental tests, several conclusions can be made :

i) with a good gains adjustment for both algorithm schemes with respect to a given input signal, the estimate of derivatives with the N2OD are less noisy than those given by the 2OD, ii) The two differentiators show good accuracy compared to the desired trajectories, iii) with the same gains tuning algorithms and when modifying the input signal, a slight degradation is observed on the estimated signals. However, the derivatives are always less noisy when using the N2OD. In order to improve the differentiators accuracy, the values of the gains must be increased. But, increasing the gain values for the 2OD tends to increase the noise amplification. In this case, the signals become unusable for control purposes. Therefore, it is worth noting that the lower noise amplification represents a real advantage for the N2OD.

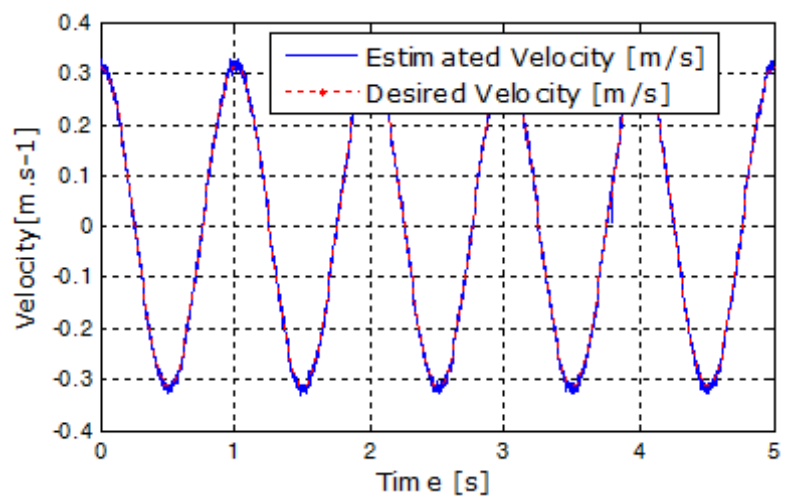

(a) Estimated velocity

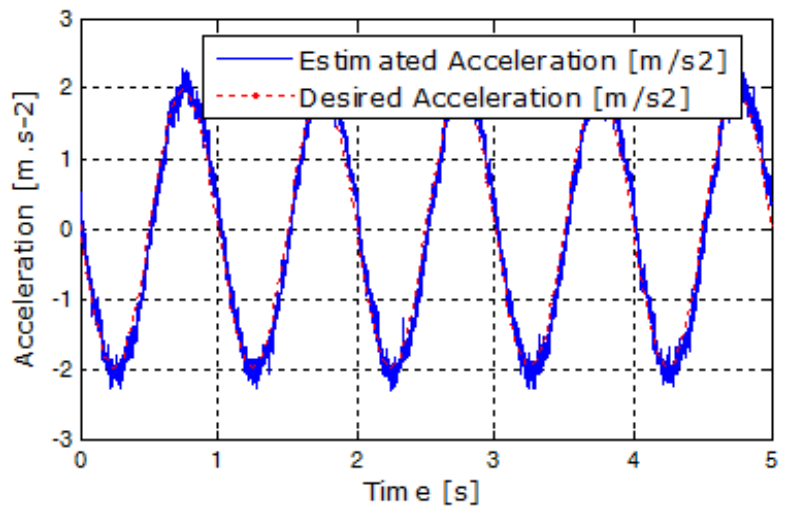

(b) Estimated acceleration

Fig. 16: Estimation velocity and acceleration: (N2OD, test 2)

\section{CONCLUSION}

In this paper, a 1st and 2nd-order sliding mode differentiators with dynamic gains have been proposed to address the problem of self-tuning gains of the classic schemes. Experimental tests are performed in the case of the position control of an electrohydraulic system in order to test the effectiveness of the proposed differentiator. These tests highlight the advantage of the new scheme which provides lower noise amplification. Indeed, the filtering property allowed by the proposed algorithm enable good accuracy to be achieved for a given spectrum of the input signal without worrying about the noise amplification on the estimated signals. From this study we can consider several further works. One is the proof of finite time convergence of the 2nd-order proposed differentiator, which it is one of the properties of the higher order sliding modes. The validation experimental of the proposed algorithm on other reference trajectories which have large frequency spectrum is also an interesting subject to be investigated. 


\section{APPENDIX}

TABLE II: Specifications of the hydraulic actuator

\begin{tabular}{|c|c|}
\hline Piston diameter & $50 \mathrm{~mm}$ \\
\hline Rod diameter & $30 \mathrm{~mm}$ \\
\hline Total moving load & $5.9 \mathrm{~kg}$ \\
\hline Length max & $166 \mathrm{~mm}$ \\
\hline Supply pressure $p_{P}$ & $210 \mathrm{bar}$ \\
\hline Maximum static force & $19858 \mathrm{~N}$ for $p_{P}=210 \mathrm{bar}$ \\
\hline Maximum dynamic force & $3238 \mathrm{~N}$ for $p_{P}=210 \mathrm{bar}$ \\
\hline
\end{tabular}

\section{REFERENCES}

[1] Merritt, H. E., Hydraulic Control Systems,John Wiley \& Sons Inc, p. 368.

[2] Re, L.D. and Isidori, A., Performances enhancement of nolinear drives by feedback linearization of linear-bilinear cascade models. Control Systems, IEEE Transactions on Control System and Technology, vol.8, 1995, pp. 1335-1345.

[3] Plummer, A.R. and Vaughan, N.D., Robust adaptive control for hydraulic servosystems, ASME Journal of Dynamics System, Measurement and Control, vol.118, 1996, pp. 237-244.

[4] Guan, C. and Pan, S., Adaptive sliding modes control of electrohydraulic system with nonlinear unknown parameters, Journal of Control Engineering Practice, vol.16, 2008, pp. 1275-1284.

[5] Yao, B., Bu, F., Reedy, J. and Chiu, G., Adaptive robsut motion control of single-rod hydraulic actuators: theory and experiments, IEEE/ASME Transactions on Mechatronics, vol.5, $n^{\circ}$ 1, 2000, pp. 79-91.

[6] Freeman, R.A and Kokotovic, P., Design of softer robust nonlinear control laws, Automatica, vol.29, 1993, pp. 1425-1437.

[7] Kanellakopoulos, I, Kokotovic, P. and Morse, A., Systematic design of adaptive controllers for feedback linearizable systems, IEEE Transactions on Automatic Control, vol.36, 1991 pp. 1241-1253.

[8] Gauthier, J. P., Hammouri, H. and Othman, S., A simple observer for nonlinear systems applications to bioreactors, IEEE Transactions on Automatic Control, vol. 37, n.37, 1992, pp. 875880.

[9] Slotine, J. E. and Li, W., Applied Nonlinear Control, Prentice-Hall, p. 461, 1991.

[10] Utkin, V.I, Guldner, J. and Shi, J. Sliding mode control in electromechanical systems, Taylor \& Francis, p. 338, 1999.

[11] Atassi, A. N. and Khalil, H. K., Separation results for the stabilization of nonlinear systems using different high-gain observer designs, Systems and Control Letters, vol. 39, 2000, pp. 183191.

[12] Levant, A., Higher order sliding modes, differentiation and output feedback control, Int. J. of Control, vol. 76, 2003, pp. 924-941.

[13] Smaoui, M., Brun, X. and Thomasset, D., A robust differentiator controller design for an electropneumatic system, Proceeding of the 44th IEEE Conference on Decision and Control, and the European Control Conference, Sevilia, Spain, 2005.

[14] Levant, A., Higher order sliding modes, Robust exact differentiation via sliding mode technique, Automatica, vol. 34, 1998, pp. 379-384.

[15] Levant, A., Sliding order and sliding accuracy in sliding mode control, International Journal of Control ,vol.58, $n^{\circ} 6$, 1993, pp. 1247-1263.

[16] Baccioti, A., Rosier, L. Lyapunov functions and satbility in control theory, SE, New York Springer-Verlag, 2005, p. 238.

[17] Clarke, F.H., Ledyaev,Y., Stern,R.J. and Wolenski, P.R. Nonsmooth analysis and control theory, New York, Springer-Verlag, 1998, p. 276.

[18] Armstrong-Helouvry, B., Dupont, P., and de Wit, C.C. A survey of models, analysis tools and compensation methods for the control of machines with friction, Automatica, vol.30, $n^{\circ} 7,1994$, pp. 1083-1138.

[19] Tustin, S. The effects of backlash and of speeddependent friction on the stability of closed-cycle controls system, Journal of the Institution of Electrical Engineers, vol. 94, $n^{\circ} 2 \mathrm{~A}, 1947$, pp. 143-151.

[20] Niksefat, N. and Sepehri, N. Design and experimental evaluation of a robust force controller for an electro-hydraulic actuator via quantitative feedback theory, Control Engineering Practice, vol. 8, 2000, pp. 1335 1345 .

[21] Guan, C. and Pan, S. Nonlinear adaptive robust control of single-rod electro-hydraulic actuator with unknown nonlinear parameters, IEEE Transactions Control Syst. Techno, vol. 16, 2008, pp. 434-445.
[22] Utkin, V.I. Nonlinear control system, 3rd Edition, Springer, 1995, p. 293.

[23] Sidhom, L. Sur les différentiateurs en temps réel: Algorithmes et Applications, PhD INSA Lyon, 2011, p. 207.

[24] Dabroom, A. and Khalil, H. K. Numerical differentiation using highgain observers, in Proc. 36th Conf. on Decision Control, San Diego, California USA, December 1997, pp. 4790-4795.

[25] Dabroom, A.M. and Khalil, H.K. Discrete-time implementation of highgain observers for numerical differentiation, Int. Journal of Control, vol. 72, no. 17, 1999, pp. 1523-1537.

[26] Brown, R.B. and Hwang, P.Y.C. Introduction to Random Signals and Applied Kalman Filtering, John Wiley Sons, 2nd ed. 1992.

[27] Davila, J., Fridman, L. and Levant, A. Second-order sliding-mode observer for mechanical systems, IEEE Transactions on Automatic Control, vol.50(11), 2005, pp. 1785-1789.

[28] Davila, J., Fridman, L., Pisanob, A. and Usaib, E. Finite-time state observation for non-linear uncertain systems via higher-order sliding modes, Int. J. of Control, vol. 82, no. 8, August 2009, pp. 1564-1574.

[29] Davila, A., Moreno, J.A., Fridman, L. Variable gains Super-Twisting algorithm : a Lypunov based design, American Control Conference, Baltimore, MD, USA, 30 June- 2 July, 2010, pp. 968-973.

[30] Moreno, J. A., Osorio, M. A Lyapunov approach to second-order sliding mode controllers and observers, Proceedings of the 47th IEEE Conference on Decision and Control, Cancun, Mexico, December 9-11, 2008.

[31] Taleb, M., Levant, A. and Plestan, F. Electropneumatic actuator control: solutions based on adaptive twisting algorithm and experimentation, Control Engineering practice, vol. 20, 2012, pp. 1-15.

[32] Plestan, F., Shtessel, Y., Bregeault, V. and Poznyak, A. Sliding mode control with gain adaptation - application to an electropneumatic actuator, Control Engineering Practice, vol. 20, June 2012.

[33] Sidhom,L., Pham, M.T., Thévenoux, F. and Gautier, M. Identification of a robot manipulator based on an adaptive higher order sliding modes differentiator, IEEE/ASME International Conference on Advanced Intelligent Mechatronics, Montreal, July 6-9, 2010.

[34] Sidhom, L., Smaoui, M.,Thomasset, D., Brun, X. and Bideaux, E. Adaptive Higher Order Sliding Modes for Two-dimensional Derivative Estimation, 18th World Congress of the International Federation of Automatic Control, Milano, 28 August - 2 September, 2011.

[35] Mboup, M., Join, C. and Fliess, M. Numerical differentiation with annihilators in noisy environnement, Numerical Algorithms, vol. 50, no. 4, 2009, pp. 439-467.

[36] Mboup, M., Join, C. and Fliess, M. A revised look at numerical differentiation with an application to nonlinear feedback control, The 15th Mediterrean Conference on Control and Automation, Athens, 2007, pp. 1-6.

[37] Alwi, H., Edwardsb, C. An adaptive sliding mode differentiator for actuator oscillatory failure case reconstruction, Automatica, vol. 49(2), no. 4, 2013, pp. 642-651.

[38] Kumar, B. Dutta Roy, S.C. Design of digital differentiators for low frequencies, Proc. IEEE, vol. 76 (3), 1988, pp. 287-289.

[39] Ovaska, S. J., Vainio, O., Laakso, T. I. Design of predictive IIR filters via feedback extension of FIR forward predictors, IEEE Trans. Instrum.Meas. vol. 46 (5), 1997, pp. 1196-1201.

[40] Proakis, J. G., Manolakis, D. G. Digital Signal Processing: Principles, Algorithms, and Applications, 3rd ed., Prentice-Hall, Upper Saddle River, NJ, 1996, Chapter 8, pp. 652-657.

[41] Soltanpour, M.R., Zolfaghari, B., Soltani, M. and Khooban, M. H. Fuzzy sliding mode control design for a class of nonlinear systems with structured and unstructured uncertainties, Int. J. of Innovative Computing, Information and Control, vol. 9, no. 7, 2013, pp. 2713-2726.

[42] Chen, F., Hou, R., Jiang, B. and Tao G. Study on fast terminal sliding mode control for a helicopter via quantum information technique and nonlinear fault observer, Int. J. of Innovative Computing, Information and Control, vol. 9, no. 8, 2013, pp. 3437-3447. 